\title{
Coloured peak algebras and Hopf algebras
}

\author{
Nantel Bergeron · Christophe Hohlweg
}

Received: 13 June 2005 / Accepted: 13 February 2006 / Published online: 11 July 2006

(C) Springer Science + Business Media, LLC 2006

\begin{abstract}
For $G$ a finite abelian group, we study the properties of general equivalence relations on $G_{n}=G^{n} \rtimes \mathfrak{S}_{n}$, the wreath product of $G$ with the symmetric group $\mathfrak{S}_{n}$, also known as the $G$-coloured symmetric group. We show that under certain conditions, some equivalence relations give rise to subalgebras of $\mathbb{k} G_{n}$ as well as graded connected Hopf subalgebras of $\bigoplus_{n \geq o} \mathbb{k} G_{n}$. In particular we construct a $G$-coloured peak subalgebra of the Mantaci-Reutenauer algebra (or $G$-coloured descent algebra). We show that the direct sum of the $G$-coloured peak algebras is a Hopf algebra. We also have similar results for a $G$-colouring of the Loday-Ronco Hopf algebras of planar binary trees. For many of the equivalence relations under study, we obtain a functor from the category of finite abelian groups to the category of graded connected Hopf algebras. We end our investigation by describing a Hopf endomorphism of the $G$-coloured descent Hopf algebra whose image is the $G$-coloured peak Hopf algebra. We outline a theory of combinatorial $G$-coloured Hopf algebra for which the $G$-coloured quasi-symmetric Hopf algebra and the graded dual to the $G$-coloured peak Hopf algebra are central objects.
\end{abstract}

Keywords Bialgebra $\cdot$ Combinatorial Hopf algebra $\cdot$ Wreath product $\cdot$ Coxeter group $\cdot$ Symmetric group $\cdot$ Hyperoctahedral group $\cdot$ Peak algebra $\cdot$ Planar binary tree $\cdot$ Descent algebra

Bergeron is partially supported by NSERC and CRC, Canada

Hohlweg is partially supported by CRC

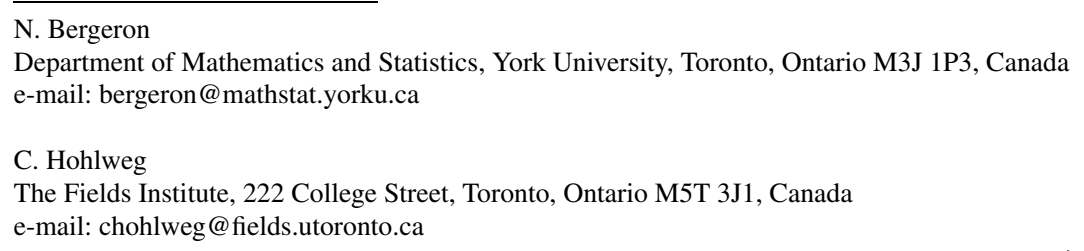




\section{Introduction}

In recent work we have seen a vivid interest in uncovering algebraic structures behind discrete objects and understanding the relationship among them. These objects are particularly interesting as their structure constants may encode various invariants in geometry, in physics or in computer science. This is particularly true in $[1,9,12$, $13,17,21,24,29]$, just to cite a few, and in [2] we find the beginning of a general theory of combinatorial Hopf algebras. Most of the algebraic structures under study are subalgebras, subcoalgebras, Hopf subalgebras or quotients of $\mathbb{k}[\mathfrak{S}]=\bigoplus_{n \geq 0} \mathbb{k} \mathfrak{S}_{n}$, where $\mathbb{k}$ is a field (of characteristic 0 ) and $\mathbb{k} \mathfrak{S}_{n}$ is the group algebra of the symmetric group $\mathfrak{S}_{n}$. There are at least two products on $\mathbb{k}[\mathfrak{S}]$. One obtained from each $\mathbb{k} \mathfrak{S}_{n}$, this is usually refer to as the (internal) homogeneous product. Malvenuto-Reutenauer [18] defines a graded Hopf structure on $\mathbb{k} \mathfrak{S}$. In particular they give an (external) graded product on $\mathbb{k} \mathfrak{S}$.

We are interested in a natural generalization of $\mathbb{k}[\mathfrak{S}]$ with similar algebraic structures. Let $G$ be a finite abelian group and let $G_{n}=G^{n} \rtimes \mathfrak{S}_{n}$ be the wreath product of $G$ with $\mathfrak{S}_{n}$; this is viewed as a $G$-colouring of $\mathfrak{S}_{n}$. Consider $\mathbb{k}[\mathrm{G}]=\bigoplus_{n \geq 0} \mathbb{k} G_{n}$. In particular, when $G=\{1\}$ we have that $\mathbb{k}[G]=\mathbb{k}[\mathfrak{S}]$. In [6], the authors extend to $\mathbb{k}[G]$ the Hopf algebra structure on $\mathbb{k}[\mathfrak{S}]$, this construction is functorial from the category of finite abelian groups to the category of graded connected Hopf algebras. It is then natural to ask if the multitude of substructures and quotients of $\mathbb{k}[\mathfrak{S}]$ studied previously can be $G$-coloured. A few answers have been given, see for instance $[1,6,15,22]$. In general this requires some work, and is not possible in all cases. Here, our approach is to study properties of equivalence relations on $\mathrm{G}=\bigoplus_{n \geq 0} G_{n}$ and to determine when they give rise to algebraic structures. Many of the algebraic objects obtained in this way are $G$-colouring of the structures in the literature, such as the peak algebras [1, 9, 21] or the Loday-Ronco Hopf algebra of trees [17]. Our work is the unifying generalization of a series of results starting in [5] and continuing in $[6,14,26]$.

In Section 1, we recall some known results about Solomon's descent algebras and Nyman's peak algebras in symmetric groups. We adopt the perspective of Atkinson [5] and Schocker [26] which is useful in subsequent sections. In Section 2 we recall the definition of the Mantaci-Reutenauer algebra [20]. These can be viewed as $G$ coloured descent algebras [6, Section 5.1]. For $G$, a finite abelian group, we develop a general study of various equivalence relations on the $G$-coloured symmetric groups. This allows us to determine which equivalence relations give rise to subalgebras and when we have a Hopf algebra structure (see Section 3). With this in hand, we show our first main theorem in Section 2.7: that the span of coloured peak elements $\mathcal{\mathcal { P }}_{n}(G)$ form a subalgebra of the Mantaci-Reutenauer algebra $\Sigma_{n}(G)$. We then have a few important theorems in Section 3 relating equivalence relations on the $G$-coloured symmetric groups and certain graded connected Hopf algebras. We also determine that under certain conditions, this map is functorial from the category of finite abelian groups to the category of graded connected Hopf algebras. This generalizes related results in [6]. We give some applications of this theory in Section 4. In particular, we describe a $G$-coloured peak Hopf algebra (a $G$-colouring of the peak Hopf algebra from [9]), and a $G$-coloured Loday-Ronco Hopf algebra (a $G$-colouring of the Loday-Ronco Hopf algebra [17]). To our knowledge, these two $G$-coloured algebras are new. We end this 
section by giving examples of application of this theory in the hyperoctahedral group $(\mathbb{Z} / 2 \mathbb{Z}$-permutations) with equivalence relations not induced from symmetric groups. Finally, in Section 5, we introduce the $\Theta_{G}$ map. This is a Hopf endomorphism of the $G$-coloured descent Hopf algebra whose image is the $G$-coloured peak Hopf algebra. We then outline a theory of combinatorial $G$-coloured Hopf algebras (generalizing [2]). We show that the Hopf algebra of $G$-coloured quasisymmetric functions (the graded dual Hopf algebra of the Mantaci-Reutenauer algebra, see [6]) is the terminal object in the category of combinatorial $G$-coloured Hopf algebras. We also state that the graded dual to the $G$-coloured peak Hopf algebra is the terminal object in the category of $o d d$ combinatorial $G$-coloured Hopf algebras.

We wish to thank Hugh Thomas for fruitful discussions and Karin Prochazka for her careful reading. The redaction of this article was completed in May 2005, when the second author was visiting the Institut Mittag-Leffler in Djursholm, Sweden.

\section{The descent algebra and the peak algebra in the symmetric group}

For $k, l \in \mathbb{Z}$, we denote by $[k, l]$ the set of integers $\{k, k+1, \ldots, l-1, l\}$ if $k \leq l$ or $\{l, l+1, \ldots, k-1, k\}$ if $l<k$.

\subsection{Words, permutations and symmetric groups}

Let us first recall some general notions about words and permutations. An alphabet $\mathcal{A}$ is a totally ordered set. Here we give the explicit description of the underlying order of $\mathcal{A}$ only when the context requires it. The elements of $\mathcal{A}$ are called letters. We denote by $\mathcal{A}^{*}$ the set of all words $w=a_{1} a_{2} \ldots a_{n}$ with letter $a_{i} \in \mathcal{A}$ and $n \geq 0$. For $n=0$ there is a unique empty word denoted by ( ). The length of a word $w=a_{1} a_{2} \ldots a_{n}$ is the integer $n$. An injective word is a word without repetition of letters. The concatenation $w \cdot w^{\prime}$ of two words $w=a_{1} \ldots a_{n}$ and $w^{\prime}=b_{1} \ldots b_{m}$ in $\mathcal{A}^{*}$ is the word $a_{1} \ldots a_{n} b_{1} \ldots b_{m}$ of length $n+m$.

There are two important ways to envision the symmetric group $\mathfrak{S}_{n}$. First as words encoding the group of bijections of the set $[1, n]$ (composing from right to left), and second as a Coxeter group. We use both points of view as needed. More specifically, let $\mathrm{N}=\{1,2,3, \ldots\}$ be the alphabet with the usual order on integers. A permutation $\sigma \in \mathfrak{S}_{n}$ is given as a word $\sigma=\sigma(1) \sigma(2) \ldots \sigma(n)$ in $\mathrm{N}^{*}$ which is injective and whose letters are in $[1, n]$. Viewed as a Coxeter group, a permutation $\sigma \in \mathfrak{S}_{n}$ is now a word in $S_{n}^{*}$ where $S_{n}=\left\{s_{1}, \ldots, s_{n-1}\right\}$ the set of simple transpositions $s_{i}=(i, i+1)$. It is well-known that $\left(\mathfrak{S}_{n}, S_{n}\right)$ is a Coxeter system of type $A_{n-1}$ (e.g. [16]). We always denote by $1_{n}$ the identity of $\mathfrak{S}_{n}$.

The length of a permutation $\sigma \in \mathfrak{S}_{n}$ is

$$
\ell(\sigma)=\mid\{(i, j) \mid i<j \in[1, n] \text { and } \sigma(i)>\sigma(j)\} \mid,
$$

namely, its number of inversions (e.g. [16]). We denote by $\omega_{n}$ the longest element in $\mathfrak{S}_{n}$. This element is an involution and $\omega_{n}(i)=n-i+1$, for all $i \in[1, n]$. 
The standardization of a word $w=a_{1} a_{2} \ldots a_{n}$ of length $n$ in $\mathcal{A}^{*}$, denoted by $\operatorname{std}(w)$, is the unique permutation $\sigma \in \mathfrak{S}_{n}$ such that for all $i<j$ we have $\sigma(i)>\sigma(j)$ if and only if $a_{i}>a_{j}$. For instance, for $w=844912$ in $\mathrm{N}^{*}$ we have $\operatorname{std}(w)=534612$.

A composition of $n$ is a word $\mathbf{c}$ in $\mathrm{N}^{*}$ whose letters sum to $n$. A composition $\mathbf{c}$ of $n$ is denoted by $\mathbf{c} \vDash n$ and we also write $n=\|\mathbf{c}\|$. To emphasize the fact that we have a composition, we write $\mathbf{c}$ as a sequence $\left(c_{1}, \ldots, c_{k}\right)$ of letter in $\mathrm{N}$ instead of a concatenation of letters. There is a well-known bijection between compositions of $n$ and subsets of $[1, n-1]$ defined by

$$
\mathbf{c}=\left(c_{1}, \ldots, c_{k}\right) \vDash n \quad \mapsto \quad I_{\mathbf{c}}=\left\{c_{1}, c_{1}+c_{2}, \ldots, c_{1}+c_{2}+\cdots+c_{k-1}\right\} .
$$

The inverse of this map is

$$
I=\left\{i_{1}, i_{2}, \ldots, i_{k}\right\} \quad \mapsto \quad \mathbf{c}_{I}=\left(i_{1}, i_{2}-i_{1}, \ldots, i_{k}-i_{k-1}, n-i_{k}\right) \vDash n .
$$

Let $\mathbf{c}=\left(c_{1}, c_{2}, \ldots, c_{k}\right) \vDash n$, we denote by $\mathfrak{S}_{\mathbf{c}}$ the subgroup of $\mathfrak{S}_{n}$ generated by $S_{\mathbf{c}}=$ $\left\{s_{i} \mid i \in[1, n] \backslash I_{\mathbf{c}}\right\}$. Such a subgroup is called a Young subgroup (or standard parabolic subgroup). Set $t_{i}=c_{1}+c_{2}+\cdots+c_{i}$ for all $i$. Given a $k$-tuple $\left(\sigma_{1}, \sigma_{2}, \ldots, \sigma_{k}\right) \in$ $\mathfrak{S}_{c_{1}} \times \mathfrak{S}_{c_{2}} \times \cdots \times \mathfrak{S}_{c_{k}}$ of permutations, we define $\sigma_{1} \times \sigma_{2} \times \cdots \times \sigma_{k} \in \mathfrak{S}_{n}$ as the permutation that maps an element $a$ belonging to the interval $\left[t_{i-1}+1, t_{i}\right]$ onto $t_{i-1}+$ $\sigma_{i}\left(a-t_{i-1}\right)$. This assignment defines an isomorphism $\mathfrak{S}_{c_{1}} \times \mathfrak{S}_{c_{2}} \times \cdots \times \mathfrak{S}_{c_{k}} \simeq \mathfrak{S}_{\mathbf{c}}$. Finally, $\omega_{\mathbf{c}}=\omega_{c_{1}} \times \cdots \times \omega_{c_{k}}$ is the longest element in $\mathfrak{S}_{\mathbf{c}}$. For instance $\omega_{(2,1,3)}=$ 213654.

For $\mathbf{c}=\left(c_{1}, \ldots, c_{k}\right)$ and $t_{i}$ as above, the subset

$$
X_{\mathbf{c}}=\left\{\sigma \in \mathfrak{S}_{n} \mid \forall i, \sigma \text { is increasing on the interval }\left[t_{i-1}+1, t_{i}\right]\right\}
$$

is a system of representatives of the left cosets of $\mathfrak{S}_{\mathbf{c}}$ in $\mathfrak{S}_{n}$. For example:

$$
X_{(2,2)}=\{1234,1324,1423,2314,2413,3412\}, \quad \text { and } \quad X_{(\underbrace{1,1, \ldots, 1}_{n \text { times }})}=\mathfrak{S}_{n} \text {. }
$$

For $\sigma \in \mathfrak{S}_{n}$, there is a unique pair $(u, v) \in X_{\mathbf{c}} \times \mathfrak{S}_{\mathbf{c}}$, called the c-components of $\sigma$, such that $\sigma=u v$. Moreover $\ell(\sigma)=\ell(u)+\ell(v)$ (see [16, Section 1.10]). Specifically, write $\sigma$ as the concatenation $\sigma_{1} \cdot \ldots \cdot \sigma_{k}$ of the words in $\mathrm{N}^{*}$ such that the length of the word $\sigma_{i}$ is $c_{i}$. It is then easy to check that

$$
v=\operatorname{std}\left(\sigma_{1}\right) \times \operatorname{std}\left(\sigma_{2}\right) \times \cdots \times \operatorname{std}\left(\sigma_{k}\right) \in \mathfrak{S}_{\mathbf{c}} \quad \text { and } \quad u=\sigma v^{-1} \in X_{\mathbf{c}} .
$$

Finally, let us recall Deodhar's Lemma for $\mathfrak{S}_{n}[11]$.

Lemma 1.1. For $\mathbf{c} \vDash n, x \in X_{\mathbf{c}}$ and $s_{i} \in S_{n}$, either

(i) $s_{i} x \in X_{\mathbf{c}}$, or

(ii) $s_{i} x=x s_{j}$, with $s_{j} \in S_{\mathbf{c}}$. In this case, $x(j)=i$ and $x(j+1)=i+1$. 
1.2. Descent sets and Solomon's descent algebra

For $\sigma \in \mathfrak{S}_{n}$, the descent set of $\sigma$ is

$$
\operatorname{Des}(\sigma)=\{i \in[1, n-1] \mid \sigma(i)>\sigma(i+1)\}
$$

The descent composition $D(\sigma)$ of $\sigma \in \mathfrak{S}_{n}$ is defined via Eq. (2): $D(\sigma)=\mathbf{c}_{\operatorname{Des}(\sigma)}$. For any $I \subseteq[1, n-1]$ we have $\operatorname{Des}\left(\omega_{\mathbf{c}_{[1, n-1] \backslash I}}\right)=I$, in particular all compositions of $n$ are descent compositions.

Denote by $\mathbb{Z} \mathfrak{S}_{n}$ the group algebra of $\mathfrak{S}_{n}$. For each $\mathbf{c} \vDash n$, we set $d_{\mathbf{c}}=\sum_{D(\sigma)=\mathbf{c}} \sigma$. Then

$$
\Sigma_{n}=\bigoplus_{\mathbf{c} \vDash n} \mathbb{Z} d_{\mathbf{c}}
$$

is a subalgebra of $\mathbb{Z} \mathfrak{S}_{n}$, called the Solomon descent algebra [27]. Let us recall the ingredients of Atkinson's [5] alternate proof of this result (see also [26, Section 4]). Let $s_{i} \in S_{n}$ and $\sigma \in \mathfrak{S}_{n}$. We define an equivalence relation as follows:

$$
\sigma \gtrless_{D} s_{i} \sigma \Longleftrightarrow \sigma^{-1} s_{i} \sigma \notin S_{n} \Longleftrightarrow\left|\sigma^{-1}(i)-\sigma^{-1}(i+1)\right|>1 \text {. }
$$

It is easily seen that $\ll_{D}$ is a symmetric relation. The reflexive and transitive closure of $\varkappa_{D}$ is called the descent equivalence relation and is denoted by $\sim_{D}$.

\section{Proposition 1.2.}

(i) For $s_{i} \in S_{n}$ and $\sigma \in \mathfrak{S}_{n}$, if $s_{i} \sigma{ }_{D} \sigma$, then $D\left(s_{i} \sigma\right)=D(\sigma)$.

(ii) For $\sigma, \tau \in \mathfrak{S}_{n}, D(\sigma)=D(\tau)$ if and only if $\sigma \sim_{D} \tau$.

Remark 1.3. This proposition was first stated by Tits [30, Theorem 2.19]tits in a more general context (see also $[5,10]$ ).

Recall that a subset $X$ of $\mathfrak{S}_{n}$ is left-connected if for each $\sigma, \tau \in X$, then there is a sequence $\sigma_{1}=\sigma, \sigma_{2}, \ldots, \sigma_{k}=\tau$ of elements in $X$ such that $\sigma_{j+1} \sigma_{j}^{-1} \in S_{n}$, for all $j \in[1, k-1]$. That is, these classes can be seen as a set of adjacent nodes in the permutohedron. In particular, Proposition 1.2 shows that the sets of permutations whose descent composition is fixed are left-connected.

Corollary 1.4. Let $I \subseteq[1, n-1]$. If there is $j \in[1, n-1]$ such that $j-1, j \in I$ and $j+1 \notin I$, then there is $\sigma \in \mathfrak{S}_{n}$ such that $\operatorname{Des}(\sigma)=I, \sigma(j)=2$ and $\sigma(j+1)=1$.

Proof: Fix $I$ and $j$ as above and consider the set

$$
T=\left\{\tau \in \mathfrak{S}_{n} \mid \operatorname{Des}(\tau)=I, \tau(j)=\tau(j+1)+1 \text {, and } \forall i<\tau(j+1), \tau^{-1}(i)<j\right\}
$$

Let $\mathbf{c}$ be such that $\operatorname{Des}\left(\omega_{\mathbf{c}}\right)=I$. Clearly $\omega_{\mathbf{c}} \in T$, so the set $T$ is nonempty. Start with any $\tau \in T$ and set $k=\tau(j+1)$. If $k=1$, then we have found the desired $\sigma=\tau$. If $k>1$, then let $\tau_{1}=s_{k-1} \tau$. Since $\tau \in T, \tau^{-1}(k-1)<j<\tau^{-1}(k)$. Therefore Eq. (5) implies that $\tau_{1} \sim_{D} \tau$. From Proposition 1.2 we have that $\operatorname{Des}\left(\tau_{1}\right)=I$. The fact that 
$j-1 \in I$ gives that $\tau_{1}^{-1}(k)=\tau^{-1}(k-1)<j-1$, so $\tau_{1}^{-1}(k+1)=\tau^{-1}(k+1)=$ $j>j-1>\tau_{1}^{-1}(k)$ and again we can apply the equivalence relation to get $\tau_{2}=$ $s_{k} \tau_{1} \sim_{D} \tau_{1} \sim_{D} \tau$. We have that $\tau_{2} \in T$ with $\tau_{2}(j+1)=k-1$. We can repeat this process $k-1$ times to get the desired $\sigma$ with $k=1$.

For $\sigma \in \mathfrak{S}_{n}$ and $\mathbf{c}, \mathbf{d}$ compositions of $n$, we define

$$
A_{\mathbf{c}, \mathbf{d}, \sigma}^{D}=\left\{(u, v) \in \mathfrak{S}_{n} \times \mathfrak{S}_{n} \mid D(u)=\mathbf{c}, D(v)=\mathbf{d}, u v=\sigma\right\} .
$$

Let us fix $s_{i} \in S_{n}$ and let $(u, v) \in \mathfrak{S}_{n} \times \mathfrak{S}_{n}$. If $u{ }^{\star}{ }_{D} s_{i} u$, we set $\psi_{i}^{D}(u, v)=\left(s_{i} u, v\right)$. If $u \varkappa_{D} s_{i} u$, then we set $\psi_{i}^{D}(u, v)=\left(u, u^{-1} s_{i} u v\right)$. Note that in the second case we have $u^{-1} s_{i} u \in S_{n}$. Since $\left(\psi_{i}^{D}\right)^{2}=\operatorname{Id}_{\mathfrak{S}_{n} \times \mathfrak{S}_{n}}$, we have that $\psi_{i}^{D}$ is a bijection.

Lemma 1.5 (Atkinson [5]). If $\sigma \in \mathfrak{S}_{n}$ and $s_{i} \in S_{n}$ are such that $\sigma \ll_{D} s_{i} \sigma$, then $\psi_{i}^{D}\left(A_{\mathbf{c}, \mathbf{d}, \sigma}^{D}\right)=A_{\mathbf{c}, \mathbf{d}, s_{i} \sigma}^{D}$, for all $\mathbf{c}, \mathbf{d}$ compositions of $n$.

Lemma 1.5 and Proposition 1.2 show that $\Sigma_{n}$ is a subalgebra of $\mathbb{Z} \mathfrak{S}_{n}$. Indeed, $\left|A_{\mathbf{c}, \mathbf{d}, \sigma}^{D}\right|$ depends only on $\mathbf{e}=D(\sigma)$. Setting $a_{\mathbf{c}, \mathbf{d}, \mathbf{e}}^{D}=\left|A_{\mathbf{c}, \mathbf{d}, \sigma}^{D}\right|$, we have

$$
d_{\mathbf{c}} d_{\mathbf{d}}=\sum_{\mathbf{e} \models n} a_{\mathbf{c}, \mathbf{d}, \mathbf{e}}^{D} d_{\mathbf{e}}
$$

Solomon's proof [27] use the basis $x_{\mathbf{c}}=\sum_{\sigma \in X_{\mathbf{c}}} \sigma$ and a study of the permutation action of $\mathfrak{S}_{n}$ on right cosets of Young subgoups (see also [7]).

\subsection{Nyman's peak algebra}

Let $\sigma \in \mathfrak{S}_{n}$, the peak set of $\sigma$ is

$$
\operatorname{peoak}(\sigma)=\{i \in[1, n-1] \mid \sigma(i-1)<\sigma(i)>\sigma(i+1), i>1\} \text {. }
$$

This set is sometimes called the interior peak set [1,3]. Contrary to descent sets, not all the subsets of $[1, n-1]$ are peak sets. In fact $I \subseteq[1, n-1]$ is a peak set if and only if $I$ satisfies the condition: if $i \in I$, then $i \geq 2$ and $i-1 \notin I$. The peak composition $P(\sigma)$ of $\sigma \in \mathfrak{S}_{n}$ is defined by Eq. (2): $P(\sigma)=\mathbf{c}_{\text {peak }(\sigma)}$. Denote by $\stackrel{\circ}{\Pi}_{n}$ the set of all compositions of $n$ which are peak compositions. From the above discussion on peak sets, it is obvious that

$$
\stackrel{\circ}{\Pi}_{n}=\left\{\left(c_{1}, \ldots, c_{k}\right) \vDash n \mid c_{i}>1 \text { for } 1 \leq i \leq k-1\right\} .
$$

Moreover, it is clear that for all $\sigma, \tau \in \mathfrak{S}_{n}$

$$
\operatorname{peoak}(\sigma) \subseteq \operatorname{Des}(\sigma) \text { and } D(\sigma)=D(\tau) \Rightarrow P(\sigma)=P(\tau)
$$


For each $\mathbf{c} \in \stackrel{\circ}{\Pi}_{n}$, we set $\stackrel{\circ}{p}_{\mathbf{c}}=\sum_{\stackrel{\circ}{P}(\sigma)=\mathbf{c}} \sigma$. Nyman $P$ [21] has shown that

$$
\stackrel{\circ}{\mathcal{P}}_{n}=\bigoplus_{\mathbf{c} \in \stackrel{\circ}{\Pi}_{n}} \mathbb{Z} \stackrel{\circ}{p}_{\mathbf{c}}
$$

is a subalgebra of $\Sigma_{n}$. We call $\stackrel{\circ}{\mathcal{P}}_{n}$ the peak algebra. We remark that $\stackrel{\circ}{\mathcal{P}}=\bigoplus_{n \geq 0} \stackrel{\circ}{\mathcal{P}}_{n}$ is also a subalgebra of the Mavenuto-Reutenauer structure on $\mathbb{k}[\mathfrak{S}]$ and that there is a

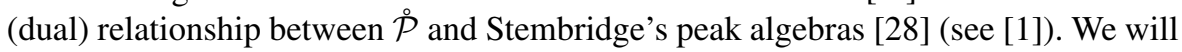
come back to this in Section 5. Here we recall Schocker's new proof [26] of Nyman's result adapting an argument of Atkinson. Let $s_{i} \in S_{n}$ and $\sigma \in \mathfrak{S}_{n}$. Then

$$
\begin{aligned}
\sigma{ }_{P} s_{i} \sigma & \Longleftrightarrow s_{i} \sigma{ }_{D} \sigma \text { or } i=1 \\
& \Longleftrightarrow\left|\sigma^{-1}(i)-\sigma^{-1}(i+1)\right|>1 \text { or } i=1 .
\end{aligned}
$$

The reflexive and transitive closure of $\approx_{P}^{\circ}$ is called the peak equivalence, and is denoted by $\sim{ }_{P}^{\circ}$.

\section{Proposition 1.6 (Schocker [26]).}

(i) For $\sigma \in \mathfrak{S}_{n}$ and $s_{i} \in S_{n}$, if $s_{i} \sigma \ll{ }_{P} \sigma$, then $P\left(s_{i} \sigma\right)=P(\sigma)$.

(ii) For $\sigma, \tau \in \mathfrak{S}_{n}, P(\sigma)=P(\tau)$ if and only if $\sigma \sim_{P}^{\circ} \tau$.

Remark 1.7. As a consequence, the sets of permutations having the same peak compositions are left-connected.

The proof of the above proposition uses Corollary 1.4. We follow a similar recipe for the proof of Proposition 4.6 in Section 4.2.

For $\sigma \in \mathfrak{S}_{n}$ and $\mathbf{c}, \mathbf{d} \in \stackrel{\circ}{\Pi}_{n}$, we set

$$
A_{\mathbf{c}, \mathbf{d}, \sigma}^{\stackrel{\circ}{P}}=\left\{(u, v) \in \mathfrak{S}_{n} \times \mathfrak{S}_{n} \mid P(u)=\mathbf{c}, P(v)=\mathbf{d}, u v=\sigma\right\} .
$$

Fix $s_{i} \in S_{n}$ and let $(u, v) \in \mathfrak{S}_{n} \times \mathfrak{S}_{n}$. Then

$$
\psi_{i}^{\stackrel{\circ}{P}}(u, v)= \begin{cases}\left(s_{1} u, v\right) & \text { if } i=1 \\ \psi_{i}^{D}(u, v) & \text { otherwise }\end{cases}
$$

is an involution on $\mathfrak{S}_{n} \times \mathfrak{S}_{n}$. The following Lemma, Eq. (7), and Proposition 1.6 show that $\stackrel{\circ}{\mathcal{P}}_{n}$ is a (non-unitary) subalgebra of $\Sigma_{n}$.

Lemma 1.8 ( Schocker [26]). If $\sigma \in \mathfrak{S}_{n}$ and $s_{i} \in S_{n}$ are such that $\sigma \varkappa_{P}^{\circ} s_{i} \sigma$, then $\psi_{i}^{\stackrel{\circ}{P}}\left(A_{\mathbf{c}, \mathbf{d}, \sigma}^{\stackrel{\circ}{P}}\right)=A_{\mathbf{c}, \mathbf{d}, s_{i} \sigma}^{\stackrel{\circ}{P}}$, for all $\mathbf{c}, \mathbf{d} \in \stackrel{\circ}{\Pi}_{n}$. 


\section{Coloured peak algebras}

\subsection{Coloured words}

Let $G$ be a finite abelian group. We denote by $1^{G}$ the identity of $G$. We also refer to $G$ as a set of colours.

Let $\mathcal{A}$ be an alphabet. We denote by $\mathcal{A}^{G}$ the alphabet $\mathcal{A} \times G$, and $a^{g}$ a letter $(a, g)$ in $\mathcal{A}^{G}$. We say that $\mathcal{A}$ is coloured by $G$. The colour of the letter $a^{g}$, denoted by $\operatorname{col}\left(a^{g}\right)$, is $g$. Let $\mathbf{g}=\left(g_{1}, \ldots, g_{n}\right)$ be a sequence of elements in $G$ and $w=a_{1} a_{2} \ldots a_{n}$ be a word in $\mathcal{A}^{*}$. We denote by $w^{\mathrm{g}}$ the word $a_{1}^{g_{1}} \cdot a_{2}^{g_{2}} \cdot \ldots \cdot a_{n}^{g_{n}}$ in $\left(\mathcal{A}^{G}\right)^{*}$. If $g_{1}=g_{2}=\cdots=$ $g_{n}=g$, we simply write $w^{\mathbf{g}}=w^{g}$. Therefore we can identify $\left(\left(\mathcal{A}^{*}\right)^{G}\right)^{*}$ and $\left(\mathcal{A}^{G}\right)^{*}$. The absolute value map $|\cdot|:\left(\mathcal{A}^{G}\right)^{*} \rightarrow \mathcal{A}^{*}$ maps a word $w^{\mathrm{g}}$ to the word $w$ in $\mathcal{A}^{*}$. This map satisfies $\left|w^{\mathbf{g}} \cdot u^{\mathbf{h}}\right|=\left|w^{\mathbf{g}}\right| \cdot\left|u^{\mathbf{h}}\right|$.

A $G$-composition $\mathbf{c}^{\mathbf{g}}$ is a word in $\left(\mathrm{N}^{G}\right)^{*}$, and $\left|\mathbf{c}^{\mathbf{g}}\right|=\mathbf{c}$ is a composition. A $G$ composition of $n$, denoted by $\mathbf{c}^{\mathbf{g}} \vDash_{G} n$, is a $G$-composition such that $\left\|\mathbf{c}^{\mathbf{g}}\right\|=\|\mathbf{c}\|=n$.

The rainbow decomposition of a word $w^{\mathrm{g}}$ in $\left(\mathcal{A}^{*}\right)^{G}$ is the unique sequence of non-empty words $w_{1}^{g_{1}}, w_{2}^{g_{2}}, \ldots, w_{k}^{g_{k}}$ such that for all $i \in[1, k-1], g_{i} \neq g_{i+1}$, and

$$
w^{\mathbf{g}}=w_{1}^{g_{1}} \cdot w_{2}^{g_{2}} \cdot \ldots \cdot w_{k}^{g_{k}}
$$

Let $c_{i}$ be the length of the word $w_{i}$. The rainbow composition of $w^{\mathbf{g}}$ is $\mathbf{r}(w)=$ $\left(c_{1}^{g_{1}}, c_{2}^{g_{2}}, \ldots, c_{k}^{g_{k}}\right)$. For instance, if $g \neq h \in G$ and $w^{\mathbf{g}}=1^{g} 4^{g} 2^{g} 6^{h} 7^{g} 3^{h} 5^{h}$, then the rainbow decomposition and the rainbow composition are

$$
w^{\mathbf{g}}=142^{g} \cdot 6^{h} \cdot 7^{g} \cdot 35^{h} \text { and } \mathbf{r}\left(w^{\mathbf{g}}\right)=\left(3^{g}, 1^{h}, 1^{g}, 2^{h}\right) .
$$

This procedure is the first part of the construction of the G-descent composition $D_{G}\left(w^{\mathbf{g}}\right)$ given by Mantaci and Reutenauer [20]. The descent composition $D(w)$ of a word in $\mathcal{A}^{*}$ is

$$
D(w)=D(\operatorname{std}(w)) .
$$

If the length of $w$ is $n$, then $D(w) \vDash n$. The $G$-descent composition $D_{G}\left(w^{\mathrm{g}}\right)$ of a word $w^{\mathbf{g}}$ in $\left(\mathcal{A}^{G}\right)^{*}$ is obtained as follows. Let $w^{\mathbf{g}}=w_{1}^{g_{1}} \cdot w_{2}^{g_{2}} \cdot \ldots \cdot w_{k}^{g_{k}}$ be the rainbow decomposition of $w^{\mathrm{g}}$. We have

$$
D_{G}\left(w^{\mathbf{g}}\right)=D\left(w_{1}\right)^{g_{1}} \cdot D\left(w_{2}\right)^{g_{2}} \cdot \ldots \cdot D\left(w_{k}\right)^{g_{k}} .
$$

Again, if the length of $w^{\mathrm{g}}$ is $n$, then $D_{G}\left(w^{\mathbf{g}}\right) \vDash_{G} n$. For instance, with $g \neq h \in G$ and $w^{\mathbf{g}}=1^{g} 4^{g} 2^{g} 6^{h} 7^{g} 3^{h} 5^{h}$, we have $D_{G}\left(w^{\mathbf{g}}\right)=\left(2^{g}, 1^{g}, 1^{h}, 1^{g}, 2^{h}\right)$.

\subsection{Coloured permutations and the Mantaci-Reutenauer algebra}

We denote by $G_{n}=G$ ? $\mathfrak{S}_{n}=G^{n} \rtimes \mathfrak{S}_{n}$ the wreath product of $G$ with $\mathfrak{S}_{n}$. An element $w \in G_{n}$ is the product $\alpha=\sigma \cdot\left(g_{1}, g_{2}, \ldots, g_{n}\right)$ where $\sigma \in \mathfrak{S}_{n}$ and $g_{i} \in G$. We call 
coloured permutations the elements of $G_{n}$. The multiplication in $G_{n}$ comes from the following commutation rule between elements of $\mathfrak{S}_{n}$ and elements of $G^{n}$ :

$$
\sigma \cdot\left(g_{1}, g_{2}, \ldots, g_{n}\right)=\left(g_{\sigma^{-1}(1)}, g_{\sigma^{-1}(2)}, \ldots, g_{\sigma^{-1}(n)}\right) \cdot \sigma .
$$

The subgroup of $G_{n}$ consisting of the element $\sigma \cdot\left(1^{G}, \ldots, 1^{G}\right)$ is isomorphic to $\mathfrak{S}_{n}$. In this case, we write $\sigma$ instead of $\sigma \cdot\left(1^{G}, \ldots, 1^{G}\right)$. Therefore $1_{n}$ is the identity of $G_{n}$. For $\mathbf{g}=\left(g_{1}, g_{2}, \ldots, g_{n}\right)$, instead of the multiplicative notation $\sigma . \mathbf{g}$, it is more convenient to use the word notation $\sigma^{\mathbf{g}}$ in $\left(\mathrm{N}^{*}\right)^{G}$.

A subset $X$ of $G_{n}$ is left-connected if for each $\alpha, \beta \in X$, there is a sequence $\alpha=$ $x_{1}, x_{2}, \ldots, x_{k}=\beta$ of elements in $X$ such that $x_{j+1} x_{j}^{-1} \in S_{n}$, for all $j \in[1, k-1]$. In particular, all the elements in a left-connected set have the same sequence of colours.

For a composition $\mathbf{c}=\left(c_{1}, c_{2}, \ldots, c_{k}\right)$ of $n$, we denote by $G_{\mathbf{c}}$ the subgroup of $G_{n}$ isomorphic to $G_{c_{1}} \times G_{c_{2}} \times \cdots G_{c_{k}}$, obtained by the map

$$
\begin{aligned}
G_{c_{1}} \times G_{c_{2}} \times \cdots G_{c_{k}} & \rightarrow G_{n} \\
\left(\sigma_{1}^{\mathbf{g}_{1}}, \sigma_{2}^{\mathbf{g}_{2}}, \ldots, \sigma_{k}^{\mathbf{g}_{k}}\right) & \mapsto\left(\sigma_{1} \times \sigma_{2} \times \cdots \times \sigma_{k}\right)^{\mathbf{g}_{1} \cdot \mathbf{g}_{2} \cdot \ldots \cdot \mathbf{g}_{k}} .
\end{aligned}
$$

For any $\alpha \in G_{n}$ we obtain a unique decomposition $\alpha=\sigma^{\mathbf{g}}=u v^{\mathbf{g}}$, where $\left(u, v^{\mathbf{g}}\right) \in$ $X_{\mathbf{c}} \times G_{\mathbf{c}}$ and $(u, v)$ are the c-components of $\sigma$ (see Section 1.1).

Denote by $\mathbb{Z} G_{n}$ the group algebra of $G_{n}$. For each $G$-composition $\mathbf{c}^{\mathrm{g}}$ of $n$, we set $d_{\mathbf{c}^{\mathrm{g}}}^{G}=\sum_{D_{G}(\alpha)=\mathbf{c}^{\mathrm{g}}} \alpha$. Then

$$
\Sigma_{n}(G)=\bigoplus_{\mathbf{c}^{\mathrm{g}} \vDash{ }_{G} n} \mathbb{Z} d_{\mathbf{c}^{\mathrm{g}}}^{G}
$$

is a subalgebra of $\mathbb{Z} G_{n}$, called the Mantaci-Reutenauer algebra [20]. It is clear, $\Sigma_{n}(\{1\})=\Sigma_{n}$. The Atkinson proof using an equivalence relation on $\mathfrak{S}_{n}$ has recently been extended to $G_{n}$ for $\Sigma_{n}(G)$ [6]. In the Sections 2.3 to 2.6, we develop a general theory of equivalence relations for the coloured symmetric groups. This will allow us to introduce coloured peak algebras in $\mathbb{Z} G_{n}$ and will be useful in Section 3 to study Hopf structures.

\subsection{Graded connected equivalence relations}

Recall that a graded set is a pair ( $E, \operatorname{deg})$ where $E$ is a set and $\operatorname{deg}: E \rightarrow \mathbb{N}$ is a map. For simplicity, we write $E$ instead of $(E, d e g)$ whenever possible. For $n \in \mathbb{N}$, we set $E_{n}=\operatorname{deg}^{-1}\{n\}$. Then

$$
\mathrm{E}=\bigoplus_{n \in \mathbb{N}} E_{n}
$$

For instance, $\mathrm{G}=\bigoplus_{n \in \mathbb{N}} G_{n}, \mathfrak{S}=\bigoplus_{n \in \mathbb{N}} \mathfrak{S}_{n}$ and $\left(\mathrm{N}^{G}\right)^{*}$ with the length of words are graded sets. On $\left(\mathrm{N}^{G}\right)^{*}$ we can also consider $\left(\left(\mathrm{N}^{G}\right)^{*},\|\cdot\|\right)$ which gives a different graded set structure. 
A graded map $\rho$ is a map $\rho: \mathrm{E}^{\prime} \rightarrow \mathrm{E}$ between two graded sets such that $\rho\left(E_{n}^{\prime}\right)$ is contained in $E_{n}$. A graded equivalence relation on a graded set $\mathrm{E}$ is an equivalence relation on each $E_{n}$. Given $\rho: \mathrm{E}^{\prime} \rightarrow \mathrm{E}$ a graded map, we define a graded equivalence relation $\sim_{\rho}$ on $\mathrm{E}^{\prime}$ where $e_{1}^{\prime} \sim_{\rho} e_{2}^{\prime}$ if and only if $\rho\left(e_{1}^{\prime}\right)=\rho\left(e_{2}^{\prime}\right)$. In this case, we can view $\rho\left(E_{n}^{\prime}\right)$ as a parametrization of the set of equivalence classes of $E_{n}^{\prime}$. Conversely, to any graded equivalence relation $\sim$ we can associate a surjective graded map $\rho$ such that $\sim$ is $\sim_{\rho}$.

Let $\rho: \mathrm{G} \rightarrow \mathrm{E}$ be a graded map and $\mathbf{c}=\left(c_{1}, \ldots, c_{k}\right) \vDash n$. We use $\rho$ to define a map on $G_{\mathbf{c}}$ as follows:

$$
\begin{aligned}
\rho_{\mathbf{c}}: G_{\mathbf{c}} & \rightarrow \underbrace{\mathrm{E} \times \mathrm{E} \times \cdots \times \mathrm{E}}_{k \text { times }} \\
v_{1} \times v_{2} \times \cdots \times v_{k} & \mapsto \rho\left(v_{1}\right) \times \rho\left(v_{2}\right) \times \cdots \times \rho\left(v_{k}\right) .
\end{aligned}
$$

It is clear that $\rho_{\mathbf{c}}=\rho_{c_{1}} \times \rho_{c_{2}} \times \cdots \times \rho_{c_{k}}$ and $\rho_{c_{i}}=\rho$ for all $i \in[1, k]$. This multiplicative notation is useful for looking at induction from Young subgroups (see Section 2.5).

A graded connected map on $\mathrm{G}$ is a graded map $\rho: \mathrm{G} \rightarrow \mathrm{E}$ where each equivalence class under $\sim_{\rho}$ is left-connected. In particular $\sim_{\rho}$ is the transitive and reflexive closure of the symmetric relation $\ll_{\rho}$ defined as follows: for $n \in \mathbb{N}, w \in G_{n}$ and $s_{i} \in S_{n}$ we have

$$
w \varkappa_{\rho} s_{i} w \Longleftrightarrow \rho(w)=\rho\left(s_{i} w\right) .
$$

In this case, we say that $\sim_{\rho}$ is a graded connected equivalence relation on $\mathrm{G}$. For instance $D, P: \mathfrak{S} \rightarrow\left(\mathrm{N}^{*},\|\cdot\|\right)$ are graded connected maps on $\mathrm{G}=\mathfrak{S}$ (when $G=\{1\}$ ).

\subsection{The induced graded connected maps $\rho_{G}$ and $\rho^{G}$}

Let $\rho: \mathfrak{S} \rightarrow$ E be a fixed graded connected map on $\mathfrak{S}$. For $G$ a finite abelian group we define a graded connected map on $\mathrm{G}$ as follow. Let $n \in \mathbb{N}, \sigma^{\mathbf{g}} \in G_{n}$ and $s_{i} \in S_{n}$. First, identify in the rainbow decomposition $\sigma^{\mathbf{g}}=\sigma_{1}^{g_{1}} \ldots \sigma_{k}^{g_{k}}$ the subword $\sigma_{\nu}$ containing $i$ as a letter and the subword $\sigma_{\mu}$ containing $i+1$ as a letter. We then define

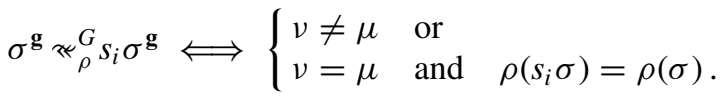

Denote by $\sim_{\rho}^{G}$ the transitive and reflexive closure of this relation. We let $E_{n}(G)=$ $G_{n} / \sim_{\rho}^{G}$ be the set of equivalence classes on $G_{n}$ and define $\rho_{G}$ the projection

$$
\rho_{G}: \mathrm{G} \rightarrow \mathrm{E}(G)=\bigoplus_{n \geq 0} E_{n}(G)
$$

This is a graded connected map on G. 
Remark 2.1. The relations $\varkappa_{D}^{\{1\}}$ and $\varkappa_{P}^{\{1\}}$ are not the relations $\varkappa_{D}$ and $\varkappa_{P}$ as defined in Section 1, but both induce the same equivalence relations. For instance $1423 \approx{ }_{P}^{\{1\}} 1432$ and $1423 \sim_{P}^{\circ} 1432$, but 1423 and 1432 are not related by $\approx_{P}^{\circ}$. More generally if $\approx_{\rho}$ is a graded symmetric relation on $\mathfrak{S}$ whose transitive and reflexive closure is $\sim_{\rho}$, we can define a symmetric graded relation $\ll_{\rho_{G}}$ on $\mathrm{G}$ as in Section 1, namely:

$$
\sigma^{\mathbf{g}}{ }_{\rho_{G}} s_{i} \sigma^{\mathbf{g}} \Longleftrightarrow \begin{cases}v \neq \mu & \text { or } \\ v=\mu & \text { and } s_{i} \sigma \nVdash_{\rho} \sigma .\end{cases}
$$

The transitive and reflexive closure of $\gtrless_{\rho_{G}}$ is precisely $\sim_{\rho}^{G}$. In fact

$$
\sigma^{\mathbf{g}} \ll_{\rho_{G}} s_{i} \sigma^{\mathbf{g}} \Rightarrow \sigma^{\mathbf{g}} \gtrless_{\rho}^{G} s_{i} \sigma^{\mathbf{g}},
$$

but the converse is not true. We will use Eq. (15) for studying general properties of graded connected maps on $\mathrm{G}$ and Eq. (17) for studying particular cases of graded connected maps like the $G$-descent composition map or the $G$-peak composition map.

We have another way to define a graded map on $\mathrm{G}$ from $\rho: \mathfrak{S} \rightarrow \mathrm{E}$. Again, let $\rho: \mathfrak{S} \rightarrow$ E be a fixed graded connected map on $\mathfrak{S}$. We first extend $\rho$ to a graded map $\mathrm{N}^{*} \rightarrow \mathrm{E}$ by setting $\rho(w)=\rho(\operatorname{std}(w))$. Then, for $\sigma^{\mathbf{g}}=\sigma_{1}^{g_{1}} \ldots \sigma_{k}^{g_{k}}$ the rainbow decomposition of $\sigma^{\mathbf{g}} \in G_{n}$ we set

$$
\rho^{G}\left(\sigma^{\mathbf{g}}\right)=\rho\left(\sigma_{1}\right)^{g_{1}} \cdot \rho\left(\sigma_{2}\right)^{g_{2}} \cdot \ldots \cdot \rho\left(\sigma_{k}\right)^{g_{k}}
$$

This defines a graded connected map $\rho^{G}: \mathrm{G} \rightarrow\left(\mathrm{E}^{G}\right)^{*}$. Observe that if $\mathrm{E}$ is the set of words $\mathcal{A}^{*}$ on an alphabet $\mathcal{A}$, then $\left(\mathrm{E}^{G}\right)^{*}=\left(\left(\mathcal{A}^{*}\right)^{G}\right)^{*}=\left(\mathcal{A}^{G}\right)^{*}$.

The graded equivalence relation $\sim{ }_{\rho^{G}}$ can be obtained as the transitive and reflective closure of the following graded connected symmetric relation $\ll_{\rho^{G}}$ on G. For this, let $\sigma^{\mathbf{g}} \in G_{n}$ and $s_{i} \in S_{n}$, and select $\mu$ and $\nu$ as in Eq. (15).

$$
\sigma^{\mathbf{g}}{ }_{\rho^{G}} s_{i} \sigma^{\mathbf{g}} \Longleftrightarrow \begin{cases}v \neq \mu & \text { or } \\ v=\mu & \text { and } \rho\left(\sigma_{v}\right)=\rho\left(s_{i} \sigma_{v}\right) .\end{cases}
$$

In general, the graded maps $\rho^{G}$ and $\rho_{G}$ have nothing in common. For instance, let $\sim_{\rho}$ be defined on $\mathfrak{S}_{n}$ by $s_{i} \sigma \varkappa_{\rho} \sigma$ if $\sigma^{-1}([i, i+1])=[1,2]$. If $g \neq h$ are two elements in $G$, then $1^{g} 2^{g} 3^{h} 4^{h} \ll_{\rho^{G}} 1^{g} 2^{g} 4^{h} 3^{h}$. Indeed, here $\sigma=1234, i=3$ and the rainbow decomposition of $\sigma^{g, g, h, h}=(12)^{g} \cdot(34)^{h}$. So $\sigma_{v}=34$ and $\rho\left(\sigma_{v}\right)=\rho(\operatorname{std}(34))=$ $\rho(12)=\rho(21)=\rho\left(\operatorname{std}\left(s_{3} 34\right)\right)=\rho\left(s_{3} 34\right)$. But these two elements are in distinct equivalence classes under $\rho_{G}$ (since $\sigma^{-1}[1,2]=[1,2] \neq[3,4]$ ):

$$
\begin{aligned}
\rho_{G}\left(1^{g} 2^{g} 3^{h} 4^{h}\right)= & \left\{1^{g} 2^{g} 3^{h} 4^{h}, 1^{g} 3^{g} 2^{h} 4^{h}, 2^{g} 3^{g} 1^{h} 4^{h}, 1^{g} 4^{g} 2^{h} 3^{h}, 2^{g} 4^{g} 1^{h} 3^{h}, 3^{g} 4^{g} 1^{h} 2^{h}\right\} \\
& \cup\left\{2^{g} 1^{g} 3^{h} 4^{h}, 3^{g} 1^{g} 2^{h} 4^{h}, 3^{g} 2^{g} 1^{h} 4^{h}, 4^{g} 1^{g} 2^{h} 3^{h}, 4^{g} 2^{g} 1^{h} 3^{h}, 4^{g} 3^{g} 1^{h} 2^{h}\right\} \\
& \text { 绝 Springer }
\end{aligned}
$$


while

$$
\begin{aligned}
\rho_{G}\left(1^{g} 2^{g} 4^{h} 3^{h}\right)= & \left\{1^{g} 2^{g} 4^{h} 3^{h}, 1^{g} 3^{g} 4^{h} 2^{h}, 2^{g} 3^{g} 4^{h} 1^{h}, 1^{g} 4^{g} 3^{h} 2^{h}, 2^{g} 4^{g} 3^{h} 1^{h}, 3^{g} 4^{g} 2^{h} 1^{h}\right\} \\
& \cup\left\{2^{g} 1^{g} 4^{h} 3^{h}, 3^{g} 1^{g} 4^{h} 2^{h}, 3^{g} 2^{g} 4^{h} 1^{h}, 4^{g} 1^{g} 3^{h} 2^{h}, 4^{g} 2^{g} 3^{h} 1^{h}, 4^{g} 3^{g} 2^{h} 1^{h}\right\}
\end{aligned}
$$

The map $\rho^{G}$ is easier to study but does not in general induce the properties we wish to extend (subalgebra, Hopf subalgebra, etc.). However, if $\rho$ possesses the induction property (Section 2.5) and the freeness property (Section 2.6), the two relations $\sim_{\rho_{G}}$ and $\sim_{\rho^{G}}$ will coincide (see Proposition 2.10).

Remark 2.2. If $\rho$ is surjective, then $\rho^{G}$ is surjective. In particular, from Eq. (18) we get a bijection

$$
\left(E^{G}\right)_{n}^{*} \rightarrow \bigoplus_{\substack{\left(c_{1}, \ldots, c_{k}\right) \models n \\ g_{i} \neq g_{i+1} \in G}} E_{c_{1}}^{g_{1}} \times \cdots \times E_{c_{k}}^{g_{k}}
$$

Thus

$$
\left|\left(E^{G}\right)_{n}^{*}\right|=|G| \sum_{\left(c_{1}, \ldots, c_{k}\right) \models n}(|G|-1)^{k-1}\left|E_{c_{1}}\right| \ldots\left|E_{c_{k}}\right| .
$$

\subsection{Induction property}

We say that a graded map $\rho: \mathfrak{S} \rightarrow \mathrm{E}$ or its graded equivalence relation $\sim_{\rho}$ have the induction property if the following condition is satisfied:

(IP) For any $n, m \in \mathbb{N}$ and $\left(e_{n}, e_{m}\right) \in E_{n} \times E_{m}$, there is $X \subseteq E_{n+m}$ such that

$$
X_{(n, m)}\left(\rho_{(n, m)}^{-1}\left(e_{n} \times e_{m}\right)\right)=\bigoplus_{e \in X} \rho^{-1}(e)
$$

where $X_{(n, m)}$ is defined in Eq. (3).

Remark 2.3. If $\rho$ has the induction property, then $\rho\left(=\rho_{n+m}\right)$ and $\rho_{(n, m)}$ coincide. More precisely, if $(u, v),\left(u^{\prime}, v^{\prime}\right) \in \mathfrak{S}_{n} \times \mathfrak{S}_{m}$ such that $\rho(u \times v)=\rho\left(u^{\prime} \times v^{\prime}\right)$, then $\rho_{(n, m)}(u \times v)=\rho_{(n, m)}\left(u^{\prime} \times v^{\prime}\right)$. The converse is not true in general.

Proposition 2.4. The graded maps $D$ and $P$ have the induction property.

Proof: For $n, m \in \mathbb{N}$, let $\mathbf{c} \models n$ and $\mathbf{d} \models m$. Let $X$ be the subset of compositions of $n+m$ such that

$$
X_{(n, m)}\left(D_{(n, m)}^{-1}(\mathbf{c} \times \mathbf{d})\right) \subseteq \bigoplus_{\mathbf{e} \in X} D^{-1}(\mathbf{e})
$$


and such that $X_{(n, m)}\left(D_{(n, m)}^{-1}(\mathbf{c} \times \mathbf{d})\right) \cap D^{-1}(\mathbf{e})$ is non empty for all $\mathbf{e} \in X$. Since $D$ is left connected and since the $(n, m)$-components are unique, to show the equality in Eq. (23) it is sufficient to prove that if $s_{i} \in S_{n+m}$ and $x(u \times v) \in X_{(n, m)}\left(D_{(n, m)}^{-1}(\mathbf{c} \times \mathbf{d})\right)$ are such that $s_{i} x(u \times v) \sim_{D} x(u \times v)$, then $s_{i} x(u \times v) \in X_{(n, m)}\left(D_{(n, m)}^{-1}(\mathbf{c} \times \mathbf{d})\right)$. Using Lemma 1.1, we reduce the problem to the case where $s_{i} x=x s_{j}$, with $j \in[1, n-1] \cup$ $[n+1, n+m-1]$. By Lemma 1.1(ii) we have

$$
1<\left|(x(u \times v))^{-1}(i)-(x(u \times v))^{-1}(i+1)\right|=\left|(u \times v)^{-1}(j)-(u \times v)^{-1}(j+1)\right| .
$$

Therefore, if $j \in[1, n-1]$, we obtain $s_{j} u \sim_{D} u$, and if $j \in[n+1, n+m-1]$, we obtain $s_{j-n} v \sim_{D} v$.

To prove that $\sim_{P}$ has the induction property we proceed as above. It is sufficient to consider the case $i=1$ in the above proof. By definition, $X_{(n, m)}$ is the set of permutations $x$ increasing on $[1, n]$ and on $[n+1, n+m]$. If $s_{1} x=x s_{j}$ we have $j=1$ or $j=n+1$ since $x(j)=1$ and $x(j+1)=2$ by Lemma 1.1(ii). In other words $s_{1} u \sim{ }_{P}^{\circ} u$ or $s_{1} v{ }_{P}^{\circ} v$.

The following lemma will be useful for Lemma 2.6.

Lemma 2.5. If $\rho$ satisfies the Condition (IP) (see Eq. (22)), then for any $\mathbf{c}=$ $\left(c_{1}, \ldots, c_{k}\right) \vDash n$ and $\left(e_{1}, \ldots, e_{k}\right) \in E_{c_{1}} \times \cdots \times E_{c_{k}}$, there is $X \subseteq E_{n}$ such that

$$
X_{\mathbf{c}}\left(\rho_{\mathbf{c}}^{-1}\left(e_{1} \times \cdots \times e_{k}\right)\right)=\bigoplus_{e \in X} \rho^{-1}(e)
$$

Proof: We proceed by induction on $k \geq 2$. The case $k=2$ is Condition (IP). Assume that $k>2$. It is well known that

$$
X_{\mathbf{c}}=X_{\left(n-c_{k}, c_{k}\right)}\left(X_{\left(c_{1}, \ldots, c_{k-1}\right)} \times 1_{c_{k}}\right)
$$

(see for instance [7, Lemma 2.1]). By the induction hypothesis, there is $X^{\prime} \subseteq E_{n-c_{k}}$ such that

$$
X_{\left(c_{1}, \ldots, c_{k-1}\right)}\left(\rho_{\left(c_{1}, \ldots, c_{k-1}\right)}^{-1}\left(e_{1} \times \cdots \times e_{k-1}\right)\right)=\bigoplus_{e^{\prime} \in X^{\prime}} \rho^{-1}\left(e^{\prime}\right) .
$$

Then by Eq. (24) we have

$$
\begin{aligned}
X_{\mathbf{c}}\left(\rho^{-1}\right. & \left.\left(e_{1} \times \cdots \times e_{k}\right)\right)=X_{\left(n-c_{k}, c_{k}\right)}\left(X_{\left(c_{1}, \ldots, c_{k-1}\right)} \times 1_{c_{k}}\right)\left(\rho_{\mathbf{c}}^{-1}\left(e_{1} \times \cdots \times e_{k}\right)\right) \\
& =X_{\left(n-c_{k}, c_{k}\right)}\left(X_{\left(c_{1}, \ldots, c_{k-1}\right)} \rho_{\left(c_{1}, \ldots, c_{k-1}\right)}^{-1}\left(e_{1} \times \cdots \times e_{k-1}\right) \times \rho^{-1}\left(e_{k}\right)\right) \\
& =X_{\left(n-c_{k}, c_{k}\right)}\left(\bigoplus_{e^{\prime} \in X^{\prime}} \rho_{\left(n-c_{k}, c_{k}\right)}^{-1}\left(e^{\prime} \times e_{k}\right)\right) \\
& =\bigoplus_{e^{\prime} \in X^{\prime}} X_{\left(n-c_{k}, c_{k}\right)}\left(\rho_{\left(n-c_{k}, c_{k}\right)}^{-1}\left(e^{\prime} \times e_{k}\right)\right) .
\end{aligned}
$$


Now by (IP) for each $e^{\prime} \in X^{\prime}$ there is $X_{e^{\prime}} \subseteq E_{n}$ such that $\rho_{\left(n-c_{k}, c_{k}\right)}^{-1}\left(e^{\prime} \times e_{k}\right)=$ $\bigoplus_{e \in X_{e^{\prime}}} \rho^{-1}(e)$. Observe that Condition (IP) forces $X_{e_{1}^{\prime}} \cap X_{e_{2}^{\prime}}=\emptyset$ if $e_{1}^{\prime} \neq e_{2}^{\prime}$. We set $X=\bigoplus_{e^{\prime} \in X^{\prime}} X_{e^{\prime}} \subseteq E_{n}$ and the proposition follows.

Lemma 2.6. If $\rho$ is a graded connected map with the induction property, then for all $\sigma^{\mathbf{g}} \in G_{n}$ and $s_{i} \in S_{n}$ we have

$$
\sigma^{\mathbf{g}}{ }_{\rho}^{G} s_{i} \sigma^{\mathbf{g}} \Rightarrow \sigma^{\mathbf{g}}{ }_{{ }_{\rho} G} s_{i} \sigma^{\mathbf{g}} \text {. }
$$

Proof: Let $\sigma^{\mathbf{g}} \in G_{n}$ and $s_{i} \in S_{n}$. Let $\sigma^{\mathbf{g}}=\sigma_{1}^{g_{1}} \ldots \sigma_{k}^{g_{k}}$ be the rainbow decomposition of $\sigma^{\mathbf{g}}$ and let $\mathbf{c}=\left(c_{1}, \ldots, c_{k}\right) \vDash n$ be such that $\mathbf{r}\left(\sigma^{\mathbf{g}}\right)=\mathbf{c}^{\mathbf{g}}$. For $(u, v) \in X_{\mathbf{c}} \times \mathfrak{S}_{\mathbf{c}}$, the c-components of $\sigma$, Eq. (4) implies that

$$
\sigma^{\mathbf{g}}=u v^{\mathbf{g}}=u\left(\operatorname{std}\left(\sigma_{1}\right)^{g_{1}} \times \cdots \times \operatorname{std}\left(\sigma_{k}\right)^{g_{k}}\right) .
$$

Identify in the rainbow decomposition the subword $\sigma_{\nu}$ containing $i$ as a letter and the subword $\sigma_{\mu}$ containing $i+1$ as a letter. Lemma 1.1 gives us that $s_{i} u \in X_{\mathbf{c}}$ if and only if $v \neq \mu$. Hence, when $v=\mu$, we obtain

$$
s_{i} \sigma^{\mathbf{g}}=u\left(\operatorname{std}\left(\sigma_{1}\right)^{g_{1}} \times \cdots \times \operatorname{std}\left(s_{i} \sigma_{v}\right)^{g_{v}} \times \cdots \times \operatorname{std}\left(\sigma_{k}\right)^{g_{k}}\right) .
$$

Comparing Eqs. (19) and (15), it is clear that the lemma follows when $v \neq \mu$. For the case $v=\mu$ and $\rho\left(s_{i} \sigma\right)=\rho(\sigma)$, since $\rho$ has the induction property, Lemma 2.5 implies

$$
\sigma, s_{i} \sigma \in X_{\mathbf{c}}\left(\rho_{\mathbf{c}}^{-1}\left(\rho_{\mathbf{c}}\left(\operatorname{std}\left(\sigma_{1}\right) \times \cdots \times \operatorname{std}\left(\sigma_{v}\right) \times \cdots \times \operatorname{std}\left(\sigma_{k}\right)\right)\right) .\right.
$$

Therefore comparing Eqs. (25) and (26) we have $\rho\left(\sigma_{v}\right)=\rho\left(s_{i} \sigma_{v}\right)$ and the lemma follows in all cases.

\subsection{Freeness property}

Now let us introduce the freeness property (the choice of this name will be explained in Corollary 3.8). We say that a graded connected map $\rho: \mathfrak{S} \rightarrow$ E or its graded equivalence relation $\sim_{\rho}$ has the freeness property if the following condition is satisfied:

(FP) For any $n, m \in \mathbb{N}, u \in X_{(n, m)}$ and $s_{j} \in S_{(n, m)}$ such that $u s_{j} u^{-1} \in S_{n+m}$.

$$
\text { If } v \in \mathfrak{S}_{(n, m)} \text { and } \rho_{(n, m)}\left(s_{j} v\right)=\rho_{(n, m)}(v) \text {, then } \rho\left(u s_{j} v\right)=\rho(u v) \text {. }
$$

We leave it to the reader to derive the following from the definitions.

Proposition 2.7. The graded connected maps $D$ and $P$ have the freeness property.

The following lemma will be useful to show the converse to Lemma 2.6. 
Lemma 2.8. If $\rho$ satisfies the freeness property, then for any $\mathbf{c}=\left(c_{1}, \ldots, c_{k}\right) \vDash n$, $u \in X_{(n, m)}$ and $s_{j} \in S_{\mathbf{c}}$ such that $u s_{j} u^{-1} \in S_{n}$, we have that $v \in \mathfrak{S}_{\mathbf{c}}$ and $\rho_{\mathrm{c}}\left(s_{j} v\right)=$ $\rho_{\mathrm{c}}(v)$ implies $\rho\left(u s_{j} v\right)=\rho(u v)$.

Proof: Let $v \in \mathfrak{S}_{\mathbf{c}}$ be such that $\rho\left(s_{j} v\right)=\rho(v)$ and write $s_{i}=u s_{j} u^{-1} \in S_{n}$. We prove that $\rho\left(u s_{j} v\right)=\rho(u v)$ by induction on $k$. The case $k=2$ is (FP). Assume that $k>2$ and write $v=v_{1} \times \cdots \times v_{k}$. Using Eq. (24) we write $u=u^{\prime}\left(u^{\prime \prime} \times 1_{c_{k}}\right)$ with $u^{\prime} \in X_{\left(n-c_{k}, c_{k}\right)}$ and $u^{\prime \prime} \in X_{\left(c_{1}, \ldots, c_{k-1}\right)}$. Since $u^{\prime} \in X_{\left(n-c_{k}, c_{k}\right)}$, Lemma 1.1 gives us that $s_{i} u^{\prime} \in X_{\left(n-c_{k}, c_{k}\right)}$ or there is $s_{p} \in S_{\left(n-c_{k}, c_{k}\right)}$ such that $s_{i} u^{\prime}=u^{\prime} s_{p}$. The second case forces $s_{p}\left(u^{\prime \prime} \times 1_{c_{k}}\right)=$ $\left(u^{\prime \prime} \times 1_{c_{k}}\right) s_{j}$. Since we have $\ell\left(s_{i} u\right)=\ell\left(u s_{j}\right)=\ell(u)+1$ and $\ell\left(u^{\prime}\left(u^{\prime \prime} \times 1_{c_{k}}\right)\right)=\ell\left(u^{\prime}\right)+$ $\ell\left(u^{\prime \prime} \times 1_{c_{k}}\right)$, the first case would imply that $\ell\left(s_{i} u^{\prime}\right)=\ell\left(u^{\prime}\right)+1$. This would imply

$$
\ell(u)=\ell\left(s_{i} u s_{j}\right)=\ell\left(s_{i} u^{\prime}\left(u^{\prime \prime} \times 1_{c_{k}}\right) s_{j}\right)=\ell\left(s_{i} u^{\prime}\right)+\ell\left(u^{\prime \prime} \times 1_{c_{k}}\right)+1>\ell(u),
$$

which is a contradiction. Hence $s_{p}=\left(u^{\prime \prime} \times 1_{c_{k}}\right) s_{j}\left(u^{\prime \prime} \times 1_{c_{k}}\right)^{-1} \in S_{\left(n-c_{k}, c_{k}\right)}$. For $s_{j} \in$ $\mathfrak{S}_{n-c_{k}}$ the induction hypothesis gives us $\rho\left(u^{\prime \prime} s_{j}\left(v_{1} \times \cdots \times v_{k-1}\right)\right)=\rho\left(u^{\prime \prime}\left(v_{1} \times \cdots \times\right.\right.$ $\left.\left.v_{k-1}\right)\right)$. The case when $s_{j} \in \mathfrak{S}_{\left(1, \ldots, 1, c_{k}\right)}$ is trivial. Then $\rho_{\mathbf{c}}\left(\left(u^{\prime \prime} \times 1_{c_{k}}\right) s_{j} v\right)=\rho_{\mathbf{c}}\left(\left(u^{\prime \prime} \times\right.\right.$ $\left.\left.1_{c_{k}}\right) v\right)$. The result follows using (FP) and the fact that $\left(u^{\prime \prime} \times 1_{c_{k}}\right) s_{j}=s_{p}\left(u^{\prime \prime} \times 1_{c_{k}}\right)$ and $s_{i} u^{\prime}=u^{\prime} s_{p}$.

Lemma 2.9. If $\rho$ is a graded connected map satisfying Condition (FP) (see Eq. (27)), then for all $\sigma^{\mathbf{g}} \in G_{n}$ and $s_{i} \in S_{n}$ we have

$$
\sigma^{\mathbf{g}}{ }_{\rho^{G}} s_{i} \sigma^{\mathbf{g}} \Rightarrow \sigma^{\mathbf{g}}{ }_{\rho}^{G} s_{i} \sigma^{\mathbf{g}} \text {. }
$$

Proof: We use the same notation as in the proof of Lemma 2.6. Again, comparing Eqs. (19) and (15), it is clear that we only have to consider the case $v=\mu$. Lemma 1.1 implies that $s_{i}=u s_{j} u^{-1}$ with $s_{j} \in S_{\mathbf{c}}$. Comparing Eqs. (25) and (26), we obtain that $\operatorname{std}\left(s_{i} \sigma_{v}\right)=s_{j_{0}} \operatorname{std}\left(\sigma_{v}\right)$ with $j_{0}=j-\left(c_{1}+\ldots+c_{v}\right)$. Then the condition $\rho\left(s_{i} \sigma_{v}\right)=\rho\left(\sigma_{v}\right)$ is equivalent to $\rho\left(s_{j_{0}} \operatorname{std}\left(\sigma_{v}\right)\right)=\rho\left(\sigma_{v}\right)$. That is $\rho_{\mathbf{c}}\left(s_{j} v\right)=\rho_{\mathbf{c}}(v)$ and the result follows from Lemma 2.8 .

Proposition 2.10. If $\rho$ is a graded connected map satisfying Conditions (IP) (see Eq. (22)) and (FP) (see Eq. (27)), then $\sim_{\rho^{G}}$ and $\gtrless_{\rho}^{G}$ are equal. In particular, the graded connected maps $\rho_{G}$ and $\rho^{G}$ induce the same equivalence relations.

For instance, consider the graded connected descent map $D_{G}$ induced from the descent (surjective) map $D: \mathfrak{S} \rightarrow \mathrm{N}^{*}$. Applying Propositions 1.2 and 2.10, we get

Corollary 2.11. For any $\alpha, \beta \in G_{n}, D_{G}(\alpha)=D_{G}(\beta)$ if and only if $\alpha \sim_{D}^{G} \beta$.

The description of $D_{G}$ given by the elementary relation $\ll_{D}^{G}$ is the principal ingredient of the proof given in [6] that $\Sigma_{n}(G)$ is a subalgebra of $\mathbb{Z} G_{n}$. 


\subsection{Coloured peak algebras}

Let $\sigma^{\mathbf{g}}=\sigma_{1}^{g_{1}} \ldots \sigma_{k}^{g_{k}}$ be the rainbow decomposition of $\sigma^{\mathbf{g}} \in G_{n}$. The G-peak composition of $\sigma^{\mathbf{g}}$ is

$$
P_{G}\left(\sigma^{\mathbf{g}}\right)=\left(P\left(\sigma_{1}\right)^{g_{1}}, \ldots, P\left(\sigma_{k}\right)^{g_{k}}\right)
$$

For instance $P_{\{g, h\}}\left(6^{g} 1^{g} 4^{g} 2^{g} 5^{h} 3^{h} 7^{h}\right)=\left(3^{g}, 1^{g}, 3^{h}\right)$. Denote by $\stackrel{\circ}{\Pi}_{n}(G)$ the set of $G$-peak compositions of $G_{n}$. Then $\left(\stackrel{\circ}{\Pi}^{G}\right)^{*}=\bigoplus_{n \in \mathbb{N}} \stackrel{\circ}{\Pi}_{n}(G)$.

In the group algebra $\mathbb{Z} G_{n}$, we form the elements ${\stackrel{\circ}{\mathbf{c}^{\mathrm{g}}}}^{G}=\sum_{\stackrel{P}{G}_{G}(\alpha)=\mathbf{c}^{\mathrm{g}}} \alpha$, where $\mathbf{c}^{\mathrm{g}} \in$ $\stackrel{\circ}{\Pi}_{n}(G)$. We then consider the subspace

$$
\stackrel{\circ}{\mathcal{P}}_{n}(G)=\bigoplus_{\mathbf{c}^{\mathbf{g}} \in \stackrel{\mathrm{\Pi}}{n}_{n}(G)} \mathbb{Z}{\stackrel{\circ}{\mathbf{c}^{\mathrm{g}}}}^{G}
$$

Theorem 2.12. If $G$ is a finite abelian group and $n \in \mathbb{N}$, then $\stackrel{\circ}{\mathcal{P}}_{n}(G)$ is a (non-unitary) subalgebra of $\Sigma_{n}(G)$ called the $G$-peak algebra.

We proceed as in Section 1.2 and the theorem will follow from the next two lemmas. First, Propositions 2.10 and Corollary 2.11, combined with Eq. (7), show that

Lemma 2.13. Let $\alpha, \beta \in G_{n}$.

(i) $\alpha \sim \underset{D}{G} \beta \Rightarrow \alpha \underset{P}{\sim_{P}^{G}} \beta$;

(ii) $P_{G}(\alpha)=P_{G}(\beta) \Longleftrightarrow \alpha \underset{P}{G} \beta$.

For $\alpha \in G_{n}$ and $\mathbf{c}^{\mathbf{g}}, \mathbf{d}^{\mathbf{h}} \in \stackrel{\circ}{\Pi}_{n}(G)$, we set

$$
A_{\mathbf{c}^{\mathbf{g}}, \mathbf{d}^{\mathbf{h}}, \alpha}^{\stackrel{\circ}{P^{\prime}}}=\left\{\left(\sigma^{\mathbf{g}}, \tau^{\mathbf{h}}\right) \in G_{n} \times G_{n} \mid P_{G}\left(\sigma^{\mathbf{g}}\right)=\mathbf{c}^{\mathbf{g}}, \quad P_{G}\left(\tau^{\mathbf{h}}\right)=\mathbf{d}^{\mathbf{h}}, \sigma^{\mathbf{g}} \tau^{\mathbf{h}}=\alpha\right\} .
$$

Fix $s_{i} \in S_{n}$ and let $\left(\sigma^{\mathbf{g}}, \tau^{\mathbf{h}}\right) \in G_{n} \times G_{n}$. Then

$$
\psi_{i}^{\stackrel{\circ}{P}_{G}}\left(\sigma^{\mathbf{g}}, \tau^{\mathbf{h}}\right)= \begin{cases}\left(s_{i} \sigma^{\mathbf{g}}, \tau^{\mathbf{h}}\right) & \text { if } s_{i} \sigma^{\mathbf{g}}{ }_{\stackrel{\circ}{P}_{G}} \sigma^{\mathbf{g}} \\ \left(\sigma^{\mathbf{g}}, \sigma^{-1} s_{i} \sigma \tau^{\mathbf{h}}\right) & \text { otherwise }\end{cases}
$$

is an involution on $G_{n} \times G_{n}$.

Lemma 2.14. If $\alpha \in G_{n}$ and $s_{i} \in S_{n}$ are such that $\alpha \lll{ }_{P}^{G} s_{i} \alpha$, then

$$
\psi_{i}^{\stackrel{\circ}{P_{G}}}\left(A_{\mathbf{c}^{\mathbf{g}}, \mathbf{d}^{\mathbf{h}}, \alpha}^{\stackrel{\circ}{\circ}}\right)=A_{\mathbf{c}^{\mathbf{g}}, \mathbf{d}^{\mathbf{h}}, s_{i} \alpha}^{\stackrel{\circ}{P^{2}}}, \quad \text { for all } \mathbf{c}^{\mathbf{g}}, \mathbf{d}^{\mathbf{h}} \in \stackrel{\circ}{\Pi}_{n}(G) .
$$

Proof: We can assume that $i$ and $i+1$ are in the same subfactor of the rainbow decomposition of $\sigma^{\mathbf{g}}$ and that $s_{i} \sigma \chi_{P} \sigma$. That is, there is $s_{j} \in S_{n}$ such that $s_{i} \sigma=\sigma s_{j}$ 祭 Springer 
and $s_{j}$ stabilize $\mathbf{g}$. Therefore $s_{i} \sigma^{\mathbf{g}}=\sigma^{\mathbf{g}} s_{j}$. If $j$ and $j+1$ are not in the same subfactor of the rainbow decomposition of $\tau^{\mathbf{h}}$ then $s_{j} \tau^{\mathbf{h}}{ }^{\star}{ }_{P_{G}} \tau^{\mathbf{h}}$ by definition. Otherwise, observe that $|\alpha|{ }_{P} s_{i}|\alpha|$ and

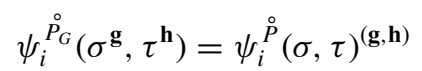

where $(u, v)^{(\mathbf{g}, \mathbf{h})}=\left(u^{\mathbf{g}}, v^{\mathbf{h}}\right)$. We conclude that $s_{j} \tau^{\mathbf{h}} \approx_{\stackrel{\circ}{P}_{G}} \tau^{\mathbf{h}}$ since $\Psi_{i}^{\stackrel{\circ}{P}}(\sigma, \tau) \in$

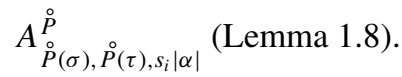

\section{Graded maps and bialgebra structures}

Henceforth we consider a field $\mathbb{k}$ of characteristic 0 .

\subsection{Hopf algebra of coloured permutations}

Let $G$ be an abelian group and recall that $\mathrm{G}=\bigoplus_{n \in \mathbb{N}} G_{n}$. Let $\rho: \mathrm{G} \rightarrow$ E be a graded map and $n \in \mathbb{N}$. We denote by $\mathcal{E}(\rho)_{n}$ the subspace of the group algebra $\mathbb{k} G_{n}$ spanned by $\mathrm{b}_{e}^{\rho}=\sum_{\rho(\alpha)=e} \alpha$ for each $e \in E_{n}$ (if $\rho^{-1}(e)=\emptyset$, we set $\mathrm{b}_{e}^{\rho}=0$ as usual). Then

$$
\mathcal{E}(\rho)=\bigoplus_{n \geq 0} \mathcal{E}(\rho)_{n}
$$

is a graded vector subspace of the graded vector space $\mathbb{k}[\mathrm{G}]=\mathcal{E}\left(\operatorname{Id}_{\mathrm{G}}\right)=\bigoplus_{n \geq 0} \mathbb{k} G_{n}$. We recall the Hopf algebra structure on $\mathbb{k}[\mathrm{G}]$ given in [6]. The product $*: \mathbb{k} G_{n} \otimes$ $\mathbb{k} G_{m} \rightarrow \mathbb{k} G_{n+m}$ is given by

$$
\alpha * \beta=x_{n, m}(\alpha \times \beta) \in \mathbb{k} G_{n+m},
$$

where $x_{n, m}=\sum_{\sigma \in X_{(n, m)}} \sigma$ (see Eq. (3)).

Let $\alpha \in G_{n}$. For each $i \in[0, n]$, we denote by $\alpha_{(i)} \times \alpha_{(n-i)}$ the unique element of $G_{i, n-i}$ such that $\left(u,\left(\alpha_{(i)} \times \alpha_{(n-i)}\right)^{-1}\right)$ are the $(i, n-i)$-components of $\alpha^{-1}$. The coproduct $\Delta: \mathbb{k}[\mathrm{G}] \rightarrow \mathbb{k}[\mathrm{G}] \otimes \mathbb{k}[\mathrm{G}]$ is the morphism of algebra (for the product $*$ ) given by

$$
\Delta(\alpha)=\sum_{i=0}^{n} \alpha_{(i)} \otimes \alpha_{(n-i)} \in \mathbb{k}[\mathrm{G}] \otimes \mathbb{k}[\mathrm{G}] .
$$

For instance, for $g \neq h$ in $G$,

$$
\begin{gathered}
1^{g} 2^{h} * 2^{h} 1^{g}=1^{g} 2^{h} 4^{h} 3^{g}+1^{g} 3^{h} 4^{h} 2^{g}+1^{g} 4^{h} 3^{h} 2^{g}+2^{g} 3^{h} 4^{h} 1^{g}+2^{g} 4^{h} 3^{h} 1^{g}+3^{g} 4^{h} 2^{h} 1^{g} ; \\
\Delta\left(2^{g} 3^{h} 1^{h} 4^{g}\right)=() \otimes 2^{g} 3^{h} 1^{h} 4^{g}+1^{h} \otimes 1^{g} 2^{h} 3^{g}+2^{g} 1^{h} \otimes 1^{h} 2^{g}+2^{g} 3^{h} 1^{h} \otimes 1^{g}+2^{g} 3^{h} 1^{h} 4^{g} \otimes() .
\end{gathered}
$$

In [6], the authors prove that $(\mathbb{k}[\mathrm{G}], *, \Delta)$ is a graded connected Hopf algebra. Moreover, the assignment $G \rightsquigarrow \mathbb{k}[\mathrm{G}]$ is a covariant functor from the category of finite abelian 
groups to the category of graded Hopf algebras. In fact the theory build in [6] is only valid for an abelian group $G$. We do not know how to do if $G$ is not abelian. The case $G=\{1\}$ is due to Malvenuto and Reutenauer [18]. Considering a finite abelian group $G$ and the morphism $G \rightarrow\{1\}$, at the level of graded Hopf algebras, we get the forgetful (surjective) map $f: \mathbb{k}[\mathrm{G}] \rightarrow \mathbb{k}[\mathfrak{S}]$ (obtained by extending linearly to $\mathbb{k}[\mathrm{G}]$ the absolute value map $||$.$) . We also have an injective morphism induced from the$ injective map $\{1\} \hookrightarrow G$.

We construct more Hopf (sub)algebras using our general theory of graded maps on G. For example:

1. The Solomon descent algebra $\Sigma=\mathcal{E}\left(D: \mathfrak{S} \rightarrow\left(\mathrm{N}^{*},\|\|.\right)\right)$ is a Hopf subalgebra of $\mathbb{k}[\mathfrak{S}][18]$.

2. The Mantaci-Reutenauer algebra $\Sigma(G)=\mathcal{E}\left(D_{G}\right)$ is a Hopf subalgebra of $\mathbb{k}[\mathrm{G}]$. Moreover, the assignment $G \rightsquigarrow \Sigma(G)$ is also a contravariant functor from the category of finite abelian groups to the category of graded Hopf algebras [6].

3. The Peak algebra $\stackrel{\circ}{\mathcal{P}}=\mathcal{E}\left(\stackrel{P}{P}: \mathfrak{S} \rightarrow\left(\mathrm{N}^{*}, \|\right.\right.$. $\left.\left.\|\right)\right)$ is a Hopf subalgebra of $\mathbb{k}[\mathfrak{S}][1,8]$.

4. The planar binary tree $\mathcal{T}(\sigma)$ of $\sigma \in \mathfrak{S}_{n}$ is defined as follows. A given $\sigma \in$ $\mathfrak{S}_{n}$ can be viewed as the concatenation of two injective words separated by the smallest letter 1: $\sigma=w^{\prime} \cdot 1 \cdot w^{\prime \prime}$. We construct $\mathcal{T}$ on permutation by induction: $\mathcal{T}(\sigma)=\mathcal{T}\left(\operatorname{std}\left(w^{\prime}\right)\right) \vee \mathcal{T}\left(\operatorname{std}\left(w^{\prime \prime}\right)\right)$. Here $T^{\prime} \vee T^{\prime \prime}$ means the grafting of $T^{\prime}$ and $T^{\prime \prime}$, see [17]. Denoting $Y_{n}$ the set of planar binary trees with $n$ vertices, we get a surjective graded map $\mathcal{T}: \mathfrak{S} \rightarrow \mathrm{Y}$, where $\mathrm{Y}=\bigoplus_{n \in \mathbb{N}} Y_{n}$. Then $\mathcal{E}(\mathcal{T})$ is the Hopf subalgebra of $\mathbb{k}[\mathfrak{S}]$, called the Loday-Ronco algebra [17].

We see that the example (2) is a $G$-coloured version of (1). In Section 4 we will present a coloured version of (3) and (4). For this, we study which properties of $\rho$ are required so that $\mathcal{E}(\rho)$ is a Hopf subalgebra.

\subsection{Induction on equivalence classes}

Let $\rho: \mathrm{G} \rightarrow \mathrm{E}$ be a graded map. The definition of the induction property (IP) (Eq. (22) in Section 2.5) can be applied to $\rho$ viewing the product in $\mathrm{G}$ instead of in S. Since the map $X_{(n, m)} \times G_{n, m} \rightarrow G_{n+m}$, sending $\left(u, v^{\mathbf{g}}\right)$ to $u v^{\mathbf{g}}$, is a bijection, we get the following:

Proposition 3.1. A graded map $\rho: \mathrm{G} \rightarrow \mathrm{E}$ satisfies Condition (IP) if and only iffor all $n, m \in \mathbb{N}$ and for all $\left(e_{1}, e_{2}\right) \in E_{n} \times E_{m}$, there is $X \subseteq E_{n+m}$ such that

$$
\mathrm{b}_{e_{1}}^{\rho} * \mathrm{~b}_{e_{2}}^{\rho}=\sum_{e \in X} \mathrm{~b}_{e}^{\rho}
$$

Corollary 3.2. Let $\rho$ be a graded map on $\mathrm{G}$. If $\rho$ has the induction property (IP), then $\mathcal{E}(\rho)$ is a subalgebra of $(\mathbb{k}[\mathrm{G}], *)$. 


\subsection{Restriction on equivalence classes}

The graded map $\rho: G \rightarrow E$ has the restriction property if it satisfies the following condition:

(RP) For any $n, m \in \mathbb{N}$ and $u \in X_{(n, m)}$, if $\alpha_{1}, \alpha_{2} \in G_{n}$ and $\beta_{1}, \beta_{2} \in G_{m}$ are such that $\rho\left(\alpha_{1}\right)=\rho\left(\alpha_{2}\right)$ and $\rho\left(\beta_{1}\right)=\rho\left(\beta_{2}\right)$, then

$$
\rho\left(\left(\alpha_{1} \times \beta_{1}\right) u^{-1}\right)=\rho\left(\left(\alpha_{2} \times \beta_{2}\right) u^{-1}\right) .
$$

Remark 3.3. If $\rho$ satisfies (RP), we obviously have:

(i) $\rho\left(\alpha_{1} \times \beta_{1}\right)=\rho\left(\alpha_{2} \times \beta_{2}\right)$, since $1_{n+m} \in X_{(n, m)}$. But we do not have that $\rho_{(n, m)}\left(\alpha_{1} \times \beta_{1}\right)=\rho_{(n, m)}\left(\alpha_{2} \times \beta_{2}\right)$; this comes from the induction property (see Remark 2.3).

(ii) For $n \in \mathbb{N}, \alpha, \beta \in G_{n}$ and $i \in[0, n]$, if $\rho\left(\alpha_{(i)}\right)=\rho\left(\beta_{(i)}\right)$ and $\rho\left(\alpha_{(n-i)}\right)=\rho\left(\beta_{(n-i)}\right)$, then $\rho\left(\alpha_{(i)} \times \alpha_{(n-i)}\right)=\rho\left(\beta_{(i)} \times \beta_{(n-i)}\right)$.

Lemma 3.4. The graded connected maps $D$ and $P$ satisfy Condition (RP).

Proof: Let $s_{i} \in S_{(n, m)}$ and $\sigma \times \tau \in \mathfrak{S}_{(n, m)}$ be such that $s_{i} \sigma \nVdash_{D} \sigma$ when $s_{i} \in S_{n}$ and $s_{i-n} \tau{ }^{\ll} \tau$ when $s_{i-n} \in S_{m}$. In both cases we have $\mid(\sigma \times \tau)^{-1}(i+1)-(\sigma \times$ $\tau)^{-1}(i) \mid>1$. For $u \in X_{(n, m)}$ we have

$\left|\left((\sigma \times \tau) u^{-1}\right)^{-1}(i+1)-\left((\sigma \times \tau) u^{-1}\right)^{-1}(i)\right|=\left|u(\sigma \times \tau)^{-1}(i+1)-u(\sigma \times \tau)^{-1}(i)\right|$

Observing that $(\sigma \times \tau)^{-1}(i+1)$ and $(\sigma \times \tau)^{-1}(i)$ are elements of either $[1, n]$ or $[n+1, n+m]$, we have that Eq. (5) holds. The lemma follows from the fact that $D$ is left connected.

For $P$ the proof is similar.

We now show that if $\rho$ has the restriction property, then $\mathcal{E}(\rho)$ is a subcoalgebra of $(\mathbb{k}[\mathrm{G}], \Delta)$. For $n \in \mathbb{N}, e \in E_{n}, i \in[0, n]$ and $\left(\beta_{1}, \beta_{2}\right) \in E_{i} \times E_{n-i}$ we set

$$
A_{\beta_{1}, \beta_{2}}^{e}=\left\{\alpha \in \rho^{-1}(e) \mid \alpha_{(i)} \times \alpha_{(n-i)}=\beta_{1} \times \beta_{2}\right\} .
$$

Lemma 3.5. Let $\rho$ be a graded map on $\mathrm{G}$ satisfying $(R P)$. For $n \in \mathbb{N}, e \in E_{n}$ and $i \in[0, n]$, if $\beta_{1}^{\prime}, \beta_{1}^{\prime \prime} \in E_{i}$ and $\beta_{2}^{\prime}, \beta_{2}^{\prime \prime} \in E_{n-i}$ are such that $\rho\left(\beta_{1}^{\prime}\right)=\rho\left(\beta_{1}^{\prime \prime}\right)$ and $\rho\left(\beta_{2}^{\prime}\right)=$ $\rho\left(\beta_{2}^{\prime \prime}\right)$, then $A_{\beta_{1}^{\prime}, \beta_{2}^{\prime}}^{e}$ and $A_{\beta_{1}^{\prime \prime}, \beta_{2}^{\prime \prime}}^{e}$ are in bijection.

Proof: If $\alpha^{\prime} \in A_{\beta_{1}^{\prime}, \beta_{2}^{\prime}}^{e}$, then $\alpha^{\prime}=\left(\beta_{1}^{\prime} \times \beta_{2}^{\prime}\right) u^{-1}$ with $u \in X_{(i, n-i)}$. Since the $(i, n-i)$ components are unique, we define a map from $A_{\beta_{1}^{\prime}, \beta_{2}^{\prime}}^{e}$ to $G_{n}$ by sending $\alpha^{\prime}$ to $\alpha^{\prime \prime}=\left(\beta_{1}^{\prime \prime} \times \beta_{2}^{\prime \prime}\right) u^{-1}$. By the restriction property, Eq. (29) and Remark 3.3, we have

$$
\rho\left(\alpha^{\prime \prime}\right)=\rho\left(\left(\beta_{1}^{\prime \prime} \times \beta_{2}^{\prime \prime}\right) u^{-1}\right)=\rho\left(\left(\beta_{1}^{\prime} \times \beta_{2}^{\prime}\right) u^{-1}\right)=\rho\left(\alpha^{\prime}\right)=e .
$$


In other words, $\alpha^{\prime \prime} \in A_{\beta_{1}^{\prime \prime}, \beta_{2}^{\prime \prime}}^{e}$. By uniqueness of the $(i, n-i)$-components again, and by symmetry of the construction, we get a bijection. If $A_{\beta_{1}^{\prime}, \beta_{2}^{\prime}}^{e}$ is empty, the above discussion shows that $A_{\beta_{1}^{\prime \prime}, \beta_{2}^{\prime \prime}}^{e}$ is also empty.

Let $e \in E_{n}$. Lemma 3.5 allows us to define for all $i \in[0, n]$ and for all $\left(e_{1}, e_{2}\right) \in$ $E_{i} \times E_{n-i}$ the integer

$$
a_{e_{1}, e_{2}}^{e}=\left|A_{\beta_{1}, \beta_{2}}^{e}\right|, \quad \text { for }\left(\beta_{1}, \beta_{2}\right) \in \rho^{-1}\left(e_{1}\right) \times \rho^{-1}\left(e_{2}\right) .
$$

Theorem 3.6. Let $\rho$ be a graded map on $\mathrm{G}$ satisfying $(R P)$ (see Eq. (28)) and $n \in \mathbb{N}$. For all $e \in E_{n}$ we have

$$
\Delta\left(\mathrm{b}_{e}^{\rho}\right)=\sum_{i=0}^{n} \sum_{\left(e_{1}, e_{2}\right) \in E_{i} \times E_{n-i}} a_{e_{1}, e_{2}}^{e} \mathrm{~b}_{e_{1}}^{\rho} \otimes \mathrm{b}_{e_{2}}^{\rho} .
$$

In particular, $\mathcal{E}(\rho)$ is a subcoalgebra of $(\mathbb{k}[\mathrm{G}], \Delta)$.

Proof: For each $i \in[0, n]$, we have

$$
\rho^{-1}(e)=\bigoplus_{\left(\beta_{1}, \beta_{2}\right) \in G_{i} \times G_{n-i}} A_{\beta_{1}, \beta_{2}}^{e}
$$

Then

$$
\begin{aligned}
\Delta\left(\mathrm{b}_{e}^{\rho}\right) & =\sum_{i=0}^{n} \sum_{\alpha \in \rho^{-1}(e)} \alpha_{(i)} \otimes \alpha_{(n-i)} \\
& =\sum_{i=0}^{n} \sum_{\left(\beta_{1}, \beta_{2}\right) \in G_{i} \times G_{n-i}} \sum_{\alpha \in A_{\beta_{1}, \beta_{2}}^{e}} \beta_{1} \otimes \beta_{2} \\
& =\sum_{i=0}^{n} \sum_{\left(e_{1}, e_{2}\right) \in E_{i} \times E_{n-i}\left(\beta_{1}, \beta_{2}\right) \in \rho^{-1}\left(e_{1}\right) \times \rho^{-1}\left(e_{2}\right)} a_{e_{1}, e_{2}}^{e} \beta_{1} \otimes \beta_{2} \\
& =\sum_{i=0}^{n} \sum_{\left(e_{1}, e_{2}\right) \in E_{i} \times E_{n-i}} a_{e_{1}, e_{2}}^{e} \mathrm{~b}_{e_{1}}^{\rho} \otimes \mathrm{b}_{e_{2}}^{\rho} .
\end{aligned}
$$

3.4. Generated connected graded maps and bialgebra structures

Recall the definition of $\rho_{G}$ in Section 2.4. The following theorem gives an automatic way to build coloured Hopf algebras.

Theorem 3.7. Let $G$ be a finite abelian group and let $\rho: \mathfrak{S} \rightarrow \mathrm{E}$ be a connected graded map.

(i) If $\rho$ has the induction property (Eq. (22)), then $\rho_{G}$ has the induction property.

(ii) If $\rho$ has the restriction property (Eq. (28)), then $\rho_{G}$ has the restriction property.

Springer 


\section{Proof:}

(i) Let $n, m \in \mathbb{N}$ and let $\left(e_{n}(G), e_{m}(G)\right) \in E_{n}(G) \times E_{m}(G)$. Let $X \subseteq E_{n+m}(G)$ be the subset such that

$$
X_{(n, m)}\left(\left(\rho_{G}\right)_{(n, m)}^{-1}\left(e_{n}(G) \times e_{m}(G)\right) \subseteq \bigoplus_{e(G) \in X} \rho_{G}^{-1}(e(G))\right.
$$

and such that $X_{(n, m)}\left(\left(\rho_{G}\right)_{(n, m)}^{-1}\left(e_{n}(G) \times e_{m}(G)\right) \cap \rho_{G}^{-1}(e(G))\right.$ is non empty for all $e(G) \in X$. Using Eq. (15), since $\rho_{G}$ is connected, to show the equality in Eq. (30) it is sufficient to prove that if $s_{i} \in S_{n+m}$ and $\sigma^{\mathbf{g}} \in X_{(n, m)}\left(\left(\rho_{G}\right)_{(n, m)}^{-1}\left(e_{n}(G) \times\right.\right.$ $\left.e_{m}(G)\right)$ are such that $s_{i} \sigma^{\mathbf{g}}{ }_{\rho}^{G} \sigma^{\mathbf{g}}$, then $s_{i} \sigma^{\mathbf{g}} \in X_{(n, m)}\left(\left(\rho_{G}\right)_{(n, m)}^{-1}\left(e_{n}(G) \times e_{m}(G)\right)\right.$. Let $\left(u, \tau_{1} \times \tau_{2}\right) \in X_{(n, m)} \times \mathfrak{S}_{(n, m)}$ be the $(n, m)$-components of $\sigma$. Then $\sigma^{\mathbf{g}}=u\left(\tau_{1} \times\right.$ $\left.\tau_{2}\right)^{\mathbf{g}_{1} \cdot \mathbf{g}_{2}}$. Using Lemma 1.1 we obtain either $s_{i} u \in X_{(n, m)}$ and the proof is done, or there is $s_{j} \in S_{(n, m)}$ such that $s_{i} u=u s_{j}$ (and $u(j)=i$ and $\left.u(j+1)=i+1\right)$. Assume first that $s_{j} \in S_{n}$. If $j$ and $j+1$ are not in the same subfactor of the rainbow decomposition of $\tau_{1}^{\mathbf{g}_{1}}$ the proof is done. If $j$ and $j+1$ are in the same subfactor then $i$ and $i+1$ are in the same subfactor of the rainbow decomposition of $\sigma^{\mathbf{g}}$ since $u(j)=i$ and $u(j+1)=i+1$. Then by definition $\rho\left(s_{i} \sigma\right)=\rho(\sigma)$. The fact that $\rho$ has the induction property forces $\rho\left(s_{j} \tau_{1}\right)=\rho\left(\tau_{1}\right)$. Hence $s_{j} \tau_{1}^{\mathbf{g}_{1}} \ll_{\rho}^{G} \tau_{1}^{\mathbf{g}_{1}}$ and the proof follows. Proceed similarly if $s_{j-n} \in S_{m}$.

(ii) Let $s_{i} \in S_{(n, m)}, \sigma^{\mathbf{g}} \times \tau^{\mathbf{h}} \in G_{n} \times G_{m}$ and $u \in X_{(n, m)}$. Observing that $i$ and $i+1$ are in the same subfactor of the rainbow decomposition of $\sigma^{\mathbf{g}} \times \tau^{\mathbf{h}}$ if and only if $i$ and $i+1$ are in the same subfactor of the rainbow decomposition of $\left(\sigma^{\mathbf{g}} \times \tau^{\mathbf{h}}\right) u^{-1}$, this case follows from definitions.

Let $\rho: \mathfrak{S} \rightarrow \mathrm{E}$ be an induced graded map satisfying (FP) (Eq. (27)) and (IP) (Eq. (22)). We have a nice description of the equivalence classes of $\rho_{G}$ by Proposition 2.10. For $g \in G$, using the map in Eq. (20), we define a monomorphism of graded vector spaces

$$
\begin{aligned}
\mu_{g}: \mathcal{E}(\rho) & \rightarrow \mathcal{E}\left(\rho_{G}\right) \\
\mathrm{b}_{e}^{\rho} & \mapsto \mathrm{b}_{e^{g}}^{\rho_{G}} .
\end{aligned}
$$

Corollary 3.8. Let $\rho: \mathfrak{S} \rightarrow \mathrm{E}$ be a graded connected map satisfying (FP) (Eq. (27)) and $(I P)(E q .(22))$. If $\mathcal{E}(\rho)$ is freely generated by $M$, then $\mathcal{E}\left(\rho_{G}\right)$ is a subalgebra of $\mathbb{k}[\mathrm{G}]$ freely generated by

$$
M(G)=\bigoplus_{g \in G} \mu_{g}(M)
$$

Proof: Observe that for $g_{1}, g_{2} \in G$ and $e_{1}, e_{2} \in \mathrm{E}$ we have

$$
\mathrm{b}_{e_{1}^{g_{1}}}^{\rho_{G}} * \mathrm{~b}_{e_{2}^{g_{2}}}^{\rho_{G}}= \begin{cases}\mathrm{b}_{e_{1}^{g_{1}} \cdot e_{2}^{g_{2}}}^{\rho_{G}} & \text { if } g_{1} \neq g_{2}, \\ \mu_{g_{1}}\left(\mathrm{~b}_{e_{1}}^{\rho} * \mathrm{~b}_{e_{2}}^{\rho}\right) & \text { if } g_{1}=g_{2} .\end{cases}
$$

Conclude by induction. 
Corollary 3.9. Let $\rho$ be a graded connected map satisfying $(F P)$ and $(I P)$. If $\mathcal{E}(\rho)$ is free, then the assignment $G \rightsquigarrow \mathcal{E}\left(\rho_{G}\right)$ is a covariant functor from the category of finite abelian groups to the category of free graded algebras.

Proof: Recall that the assignment $G \rightsquigarrow \mathbb{k}[\mathrm{G}]$ is a covariant functor from the category of finite abelian groups to the category of graded Hopf algebras [6]. More precisely if $f: G \rightarrow G^{\prime}$ is a group homomorphism, we define a Hopf algebra homomorphism $f_{*}$ by sending $\sigma^{\mathbf{g}} \in \mathbb{k}[\mathrm{G}]$ to $\sigma^{f(\mathbf{g})} \in \mathbb{k}\left[\mathrm{G}^{\prime}\right]$ (where $f\left(\left(g_{1}, \ldots, g_{n}\right)\right)=\left(f\left(g_{1}\right), \ldots, f\left(g_{n}\right)\right)$ ). Then we have $f_{*} \circ \mu_{g}=\mu_{f(g)}$ for all $g \in G$. In other words, $f_{*}(M(G)) \subseteq M\left(G^{\prime}\right)$. The corollary follows.

\section{Applications}

4.1. Coloured bialgebras of peaks and trees

A first application of the above theory is the following theorem:

Theorem 4.1. Let $G$ be an abelian group.

(i) The graded space

$$
\stackrel{\circ}{\mathcal{P}}(G)=\bigoplus_{n \in \mathbb{N}} \stackrel{\circ}{\mathcal{P}}_{n}(G)
$$

is a subalgebra of $(\Sigma(G), *)$ freely generated by

$$
\left\{\stackrel{\circ}{p}_{(n)^{g}}^{G} \mid g \in G, n \text { odd }\right\} .
$$

(ii) The graded algebra $\stackrel{\mathcal{P}}{(}(G)$ is a Hopf subalgebra of $(\Sigma(G), *, \Delta)$. Moreover, the assignment $G \rightsquigarrow \stackrel{\mathcal{P}}{ }(G)$ is a covariant functor from the category of finite abelian groups to the category of graded connected Hopf algebras.

Proof: The fact that $\stackrel{\circ}{\mathcal{P}}(G)$ is a Hopf subalgebra of $(\Sigma(G), *, \Delta)$ comes from Propositions 2.4 and 3.4, Theorems 3.6 and 3.7, and Corollary 3.2. The peak algebra $\stackrel{\circ}{\mathcal{P}}$ is freely generated by $\stackrel{\circ}{p}_{(n)}$ for $n$ odd [9, Theorem 5.4]. Then the freeness follows from Corollary 3.8, and the functorial property follows from Corollary 3.9.

We next consider a $G$-colouring of the Loday-Ronco Hopf algebra of trees [17]. Let $\sigma^{\mathbf{g}}=\sigma_{1}^{g_{1}} \ldots \sigma_{k}^{g_{k}}$ be the rainbow decomposition of $\sigma^{\mathbf{g}} \in G_{n}$. The $G$-sequence of trees of $\sigma^{\mathbf{g}}$ is

$$
\mathcal{T}_{G}\left(\sigma^{\mathbf{g}}\right)=\left(\mathcal{T}\left(\operatorname{std}\left(\sigma_{1}\right)\right)^{g_{1}}, \ldots, \mathcal{T}\left(\operatorname{std}\left(\sigma_{k}\right)\right)^{g_{k}}\right)
$$


(see Section 3.1, Example 4). For example if $g, h, k$ are three distinct elements of $G$ then $\mathcal{T}_{G}\left(5^{g} 3^{g} 6^{g} 1^{h} 4^{h} 2^{k}\right)=\left(\mathcal{T}(213)^{g}, \mathcal{T}(12)^{h},(1)^{k}\right)$ which gives

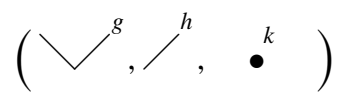

Hivert, Novelli and Thibon [12-14] have shown that the graded map $\mathcal{T}$ is connected. The dual-sylvester equivalence is defined by

$$
s_{i} \sigma{ }_{\operatorname{sylv}^{*}} \sigma \Longleftrightarrow \exists k \in\left[w^{-1}(i), w^{-1}(i+1)\right], \sigma(k)>i+1
$$

They also show that $\mathcal{T}(w)=\mathcal{T}(g) \Longleftrightarrow w \sim_{\text {sylv }^{*}} g$. The following lemma has been indirectly proved in [14].

Lemma 4.2. The graded connected map $\mathcal{T}$ satisfies (FP) (Eq. (27)), (IP) (Eq. (22)) and $(R P)(E q .(28))$.

Proof: Proceed as in Propositions 2.4, 2.7 and 3.4.

Theorem 4.3. The graded space $\mathcal{E}\left(\mathcal{T}_{G}\right)$ is a graded connected Hopf subalgebra of $\mathbb{k}[\mathrm{G}]$ containing $\Sigma(G)$. As an algebra, it is freely generated by

$$
\left\{\mathrm{b}_{(\mid \vee T)^{g}}^{\mathcal{T}_{G}} \mid g \in G, T \in Y_{n-1}\right\},
$$

where $\mid \in Y_{1}$ is the unique tree of degree 1 and $\vee$ is the grafting operation (see [17]). Moreover, the assignment $G \rightsquigarrow \mathcal{E}\left(\mathcal{T}_{G}\right)$ is a covariant functor from the category of finite abelian groups to the category of graded connected Hopf algebras.

Proof: The fact that $\mathcal{E}\left(\mathcal{T}_{G}\right)$ is a Hopf subalgebra of $(\mathbb{k}[\mathrm{G}], *, \Delta)$ comes from Lemma 4.2, Theorems 3.6 and 3.7, and Corollary 3.2. The Loday-Ronco algebra of trees $\mathcal{E}(\mathcal{T})$ is freely generated by $\mathrm{b}_{\mid \vee T}^{\mathcal{T}}$ for $T \in Y_{n-1}[17$, Theorem 3.8]. The freeness follows from Corollary 3.8, and the functorial property follows from Corollary 3.9.

Remark 4.4. We could also apply this theory to the Knuth relations and the Hopf algebra of tableaux [23]. This would lead to a different $G$-coloured version than the one defined in [6, Section 5.5].

4.2. Exterior peaks in the symmetric group

Let $\sigma \in \mathfrak{S}_{n}$, the set of exterior peaks of $\sigma$ is

$$
\operatorname{peak}(\sigma)=\{i \in[1, n-1] \mid \sigma(i-1)<\sigma(i)>\sigma(i+1)\}
$$

where we set $\sigma(0)=0$ [1, Definition 3.1]. Notice that $1 \in \operatorname{peak}(\sigma)$ if and only if $1 \in \operatorname{Des}(\sigma)$. Similarly to peak sets, not all the subsets of $[1, n-1]$ are exterior peak 
sets. In fact $I \subseteq[1, n-1]$ is an exterior peak set if and only if $I$ satisfies the condition: if $i \in I \backslash\{1\}$, then $i-1 \notin I$. The peak composition $P(\sigma)$ of $\sigma \in \mathfrak{S}_{n}$ is defined by Eq. (2): $P(\sigma)=\mathbf{c}_{\text {peak }(\sigma)}$. Denote by $\Pi_{n}$ the set of all compositions of $n$ which are peak compositions. From the above discussion on sets of peaks, it is obvious that

$$
\Pi_{n}=\left\{\mathbf{c}=\left(c_{1}, \ldots, c_{k}\right) \vDash n \mid c_{i}>1,2 \leq i \leq k-1\right\} .
$$

Moreover, it is clear that for all $\sigma, \tau \in \mathfrak{S}_{n}$ we have peak $(\sigma) \subseteq \operatorname{peak}(\sigma) \subseteq \operatorname{Des}(\sigma)$ and $D(\sigma)=D(\tau) \Rightarrow P(\sigma)=P(\tau) \Rightarrow P(\sigma)=P(\tau)$.

For each $\mathbf{c} \in \Pi_{n}$, we set $p_{\mathbf{c}}=\sum_{P(\sigma)=\mathbf{c}} \sigma$. Then Aguiar, Bergeron and Nyman [1] have shown that

$$
\mathcal{P}_{n}=\bigoplus_{\mathbf{c} \in \Pi_{n}} \mathbb{Z} p_{\mathbf{c}}
$$

is a subalgebra of $\Sigma_{n}$. We call $\mathcal{P}_{n}$ the exterior peak algebra. They have also shown that the graded space $\mathcal{P}=\mathcal{E}(P)$ is a subcoalgebra of $(\mathbb{k}[\mathfrak{S}], \Delta)$.

In [26], Schocker has given an analog of Atkinson's proof of Solomon's result for the peak algebra (see Section 1.3). Here we give a similar proof for the exterior peak algebra. For $s_{i} \in S_{n}$ and $\sigma \in \mathfrak{S}_{n}$, we define the exterior peak equivalence relation as follows:

$$
\begin{aligned}
\sigma \aleph_{P} s_{i} \sigma & \Longleftrightarrow\left\{\begin{array}{l}
s_{i} \sigma \aleph_{D} \sigma \text { or } \\
i=1 \text { and } s_{1} \sigma \neq \sigma s_{1},
\end{array}\right. \\
& \Longleftrightarrow \begin{cases}\left|\sigma^{-1}(i)-\sigma^{-1}(i+1)\right|>1 \text { or } \\
i=1 \text { and }[\sigma(1), \sigma(2)] \neq[1,2] .\end{cases}
\end{aligned}
$$

It is easily seen that $\varkappa_{P}$ is a symmetric relation. The reflexive and transitive closure of $\varkappa_{P}$ is called the exterior peak equivalence, and is denoted by $\sim_{P}$.

Lemma 4.5. For $\sigma \in \mathfrak{S}_{n}$ and $s_{i} \in S_{n}$, if $s_{i} \sigma \ll_{P} \sigma$, then $P\left(s_{i} \sigma\right)=P(\sigma)$.

Proof: By Proposition 1.2 and Eq. (32) we can easily reduce the proof to the case where $i=1$, the letters 1,2 are adjacent and $[\sigma(1), \sigma(2)] \neq[1,2]$. As the word $1 \cdot 2$ (or 2 . 1) is not a prefix of the word $\sigma$, it is obvious that exchanging 1 and 2 does not change peak $(\sigma)$.

Proposition 4.6. For $\sigma, \tau \in \mathfrak{S}_{n}, P(\sigma)=P(\tau)$ if and only if $\sigma \sim_{P} \tau$.

Proof: By Lemma 4.5 we have only to show that if $P(\sigma)=P(\tau)$ then $\sigma \sim_{P} \tau$.

Assume that $I=\operatorname{Des}(\sigma) \neq \operatorname{peak}(\sigma)$, then there is $j \in I$ such that $j, j-1 \in I$ and $j+1 \notin I$. Let $\sigma^{\prime} \in \mathfrak{S}_{n}$ be such that $\operatorname{Des}\left(\sigma^{\prime}\right)=I$ and such that $\sigma^{\prime}(j)=2=\sigma^{\prime}(j+$ $1)+1$ (Corollary 1.4). We have $s_{1} \sigma^{\prime} \ll_{P} \sigma^{\prime}$, that is $P(\sigma)=P\left(s_{1} \sigma^{\prime}\right)$ and $\left|\operatorname{Des}\left(s_{1} \sigma^{\prime}\right)\right|=$ $\left|\operatorname{Des}\left(\sigma^{\prime}\right)\right|-1$. By induction on $\operatorname{Des}(\sigma)$ and by Proposition 1.2(ii) we obtain an element $\sigma_{1} \in \mathfrak{S}_{n}$ such that $\sigma \sim_{D} \sigma^{\prime} \sim_{P} \sigma_{1}$ and $\operatorname{Des}\left(\sigma_{1}\right)=\operatorname{peak}\left(\sigma_{1}\right)=\operatorname{peak}(\sigma)$. In other words $\sigma \sim_{P} \sigma_{1}$ since two permutations having the same descent sets have the same exterior 祭 Springer 
peak set. Proceed similarly with $\tau$ to obtain a permutation $\tau_{1}$ such that $\operatorname{Des}\left(\tau_{1}\right)=$ $\operatorname{peak}\left(\tau_{1}\right)=\operatorname{peak}(\tau)$. Then $\tau \sim_{P} \tau_{1}$. As $\operatorname{Des}\left(\tau_{1}\right)=\operatorname{Des}\left(\sigma_{1}\right)$ and as $\tau_{1} \sim_{D} \sigma_{1}$ implies $\tau_{1} \sim_{P} \sigma_{1}$ the proposition follows from Proposition 1.2.

The following corollary is immediate.

Corollary 4.7. Each equivalence class under $\sim_{P}$ is left-connected.

If $\sigma \in \mathfrak{S}_{n}$ and $\mathbf{c}, \mathbf{d} \in \Pi_{n}$, we set

$$
A_{\mathbf{c}, \mathbf{d}, \sigma}^{P}=\left\{(u, v) \in \mathfrak{S}_{n} \times \mathfrak{S}_{n} \mid P(u)=\mathbf{c}, P(v)=\mathbf{d}, u v=\sigma\right\} .
$$

For $s_{i} \in S_{n}$ and $(u, v) \in \mathfrak{S}_{n} \times \mathfrak{S}_{n}$, we define

$$
\psi_{i}^{P}(u, v)= \begin{cases}\psi_{i}^{D}(u, v) & \text { if } s_{i} u \ll_{D} u \\ \left(s_{1} u, v\right) & \text { if } i=1 \text { and } s_{1} u \neq u s_{1} \\ \left(u, s_{1} v\right) & \text { if } i=1 \text { and } s_{1} u=u s_{1}\end{cases}
$$

We have $\left(\psi_{i}^{P}\right)^{2}=\operatorname{Id}_{\mathfrak{S}_{n} \times \mathfrak{S}_{n}}$. In particular $\psi_{i}^{P}$ is a bijection.

Lemma 4.8. If $\sigma \in \mathfrak{S}_{n}$ and $s_{i} \in S_{n}$ are such that $\sigma \ll_{P} s_{i} \sigma$, then

$$
\psi_{i}^{P}\left(A_{\mathbf{c}, \mathbf{d}, \sigma}^{P}\right)=A_{\mathbf{c}, \mathbf{d}, s_{i} \sigma}^{P}, \quad \text { for all } \mathbf{c}, \mathbf{d} \in \Pi_{n} .
$$

Proof: Let $(u, v) \in A_{\mathbf{c}, \mathbf{d}, \sigma}^{P}$. First assume that $\sigma \varkappa_{P} s_{1} \sigma$. Observe that if $s_{1} u=u s_{1}$ and $s_{1} v=v s_{1}$, then $s_{1} \sigma=\sigma s_{1}$ which is a contradiction. In this case $\psi_{i}^{P}(u, v) \in A_{\mathbf{c}, \mathbf{d}, s_{i} \sigma}^{P}$. Assume next that $\sigma{ }_{D} s_{i} \sigma$. We observe from Lemma 1.5 and peak $(\sigma) \subseteq \operatorname{Des}(\sigma)$ that if $s_{i} u{ }_{D} u$, then

$$
\psi_{i}^{P}(u, v)=\psi_{i}^{D}(u, v) \in \psi_{i}^{D}\left(A_{D(u), D(v), \sigma}^{D}\right)=A_{D(u), D(v), s_{i} \sigma}^{D} \subseteq A_{\mathbf{c}, \mathbf{d}, s_{i} \sigma}^{P} .
$$

Lemma 4.9. The graded connected map P has the restriction property (Eq. (28)).

Proof: Proceed as in the proof of Lemma 3.4.

Remark 4.10. The graded map $P$ does not have the induction property. For example, the set $X_{(1,2)}(\{1\} \times\{12\})=\{123,213,312\}$ does not contain $321 \varkappa_{P} 312$.

Then Lemmas 4.8 and 4.9, Proposition 4.6 and Theorem 3.6 imply directly the following theorem.

\section{Theorem 4.11.}

(i) $\mathcal{P}_{n}$ is a subalgebra of $\Sigma_{n}$.

(ii) $\mathcal{P}$ is a subcoalgebra of $(\mathbb{k}[\mathfrak{S}], \Delta)$. 
In general the graded connected map $P^{G}$ defined by Eq. (18) does not have the induction property nor the restriction property.

\subsection{Coalgebras in the hyperoctahedral group}

We give here some examples of coalgebras associated to graded map which are not induced from graded maps on the symmetric group.

For $G=\mathbb{Z} / 2 \mathbb{Z}=\{-1,+1\}$, the hyperoctahedral group $G_{n}$ is a Coxeter group of type $B_{n}$ generated by $S_{n}^{ \pm}=\left\{s_{0}, s_{1}, \ldots, s_{n-1}\right\}=\left\{s_{0}\right\} \cup S_{n}$ where $s_{0}=1_{n}^{(-1,+1,+1, \ldots,+1)}$. The Coxeter length of $\alpha \in G_{n}$ is in this case

$$
\ell_{B}(\alpha)=\min \left\{k>0 \mid \alpha=r_{1} \ldots r_{k}, r_{i} \in S_{n}^{ \pm}\right\} .
$$

Recall that a subset $X$ of $G_{n}$ is left-B-connected if for each $\alpha, \beta \in X$, there is a sequence $\alpha=\alpha_{1}, \alpha_{2}, \ldots, \alpha_{k}=\beta$ of elements in $X$ such that $\alpha_{j+1} \alpha_{j}^{-1} \in S_{n}^{ \pm}$, for all $j \in[1, k-1]$. That is, these classes can be seen as a set of adjacent nodes in the type B-permutahedron.

The B-descent set of $\alpha \in G_{n}$, with the convention that $\alpha(0)=0$, is

$$
\begin{aligned}
\operatorname{Des}_{B}(\alpha) & =\left\{i \in[0, n-1] \mid \ell_{B}\left(\alpha s_{i}\right)<\ell_{B}(\alpha)\right\} \\
& =\{i \in[0, n-1] \mid \alpha(i)>\alpha(i+1)\}
\end{aligned}
$$

We get a graded map $\operatorname{Des}_{B}: G \rightarrow \bigoplus_{n \in \mathbb{N}}\{$ subsets of $[0, n-1]\}$ and $\mathcal{E}\left(\operatorname{Des}_{B}\right)_{n}$ is precisely the Solomon descent algebra $\Sigma\left(G_{n}\right)$ associated to $G_{n}$ [27]. Atkinson [5] has shown an analog of Proposition 1.2 for B-descent sets: B-descent sets are left-Bconnected. More precisely, the type B descent equivalence is defined as the transitive and reflexive closure of the following relation: if $r \in S_{n}^{ \pm}$and $\alpha \in G_{n}$, then

$$
r \alpha \ll_{\operatorname{Des}_{B}} \alpha \Longleftrightarrow \alpha^{-1} r \alpha \notin S_{n}^{ \pm}
$$

or equivalently

$$
r \alpha \ll_{\operatorname{Des}_{B}} \alpha \Longleftrightarrow\left\{\begin{array}{lll}
r \in S_{n} & \text { and } & r \alpha \ll_{D}^{G} \alpha \\
r=s_{0} & \text { and } & |\alpha(1)|>1
\end{array}\right.
$$

since $s_{0}$ is not in the conjugacy class of $s_{i} \in S_{n}$. Obviously $\alpha \sim{ }_{D}^{G} \beta \Rightarrow \alpha \sim_{\operatorname{Des}_{B}} \beta$. Hence $\Sigma\left(G_{n}\right) \subseteq \Sigma_{n}(G)$.

In [1,9], the authors have shown that $\Sigma_{B}=\mathcal{E}\left(\operatorname{Des}_{B}\right)$ is a subcoalgebra of $(\Sigma(G), \Delta)$. With our theory we obtain this result as a consequence of the following lemma.

Lemma 4.12. The graded map $\operatorname{Des}_{B}$ has the restriction property.

Proof: Let $n, m \in \mathbb{N}$ and $u \in X_{(n, m)}$. For $\alpha \in G_{n}$ and $\beta \in G_{m}$, we have to show that for $r \in S_{n}^{ \pm}$, either of the following holds:

(i) if $r \alpha \ll_{\operatorname{Des}_{B}} \alpha$, then $(r \alpha \times \beta) u^{-1} \ll_{\operatorname{Des}_{B}}(\alpha \times \beta) u^{-1}$,

(ii) if $r \beta \ll_{\operatorname{Des}_{B}} \beta$, then $\operatorname{Des}_{B}\left((\alpha \times r \beta) u^{-1}\right)=\operatorname{Des}_{B}\left((\alpha \times \beta) u^{-1}\right)$. 
By Eq. (35) we have to consider only $r=s_{0}$, since Theorem 3.7 gives us that $D_{G}$ has the restriction property. It is well known that $\mathfrak{S}_{(n . m)}$ is a parabolic subgroup of $G_{n+m}$ and that $\ell_{B}\left(\left(v_{1} \times v_{2}\right) u^{-1}\right)=\ell_{B}\left(v_{1} \times v_{2}\right)+\ell_{B}\left(u^{-1}\right)$. The lemma follows easily using Eq. (34).

Remark 4.13. In symmetric groups the graded map $\mathcal{T}$ gives us a construction of the associahedron from the permutohedron (see for instance [17]). For the hyperoctahedral groups, such a map has been described by Reiner [25]. Let us denote by $\mathrm{Gon}_{n}$ the set of centrally symmetric $(2 n+2)$-gons and let

$$
\mathcal{G}: \mathrm{G} \rightarrow \mathrm{Gon}=\bigoplus_{n \in \mathbb{N}} \mathrm{Gon}_{n}
$$

be the graded map given by Reiner. This map gives a construction of the cyclohedron from the type $B$-permutohedron. It is a left-B-connected graded map. In a work in progress [4], Aguiar and Thomas have observed that $\mathcal{E}(\mathcal{G})$ is a subcoalgebra of $(\mathbb{k}[G], \Delta)$. Recently, Reading [24] defined a family of $G$-equivalences relations, one for each orientation of the Coxeter diagram, whose equivalence classes are parameterized by the centrally symmetric $(2 n+2)$-gons. We could prove using the relation arising from the left-to-right orientation that $\mathcal{G}$ has the restriction property, and then, we could obtain another proof of the above result of Aguiar and Thomas. We may check that except for the relation arising from the righ-to-left orientation, none of the others have the restriction property. It is interesting to notice that the number of centrally symmetric $(2 n+2)$-gons is $\left(\begin{array}{c}2 n \\ n\end{array}\right)$ which is also the number of $G$-sequences of trees whose sum of vertices is $n$ (see Eq. (21)). That means that the dimension of homogeneous parts of $\mathcal{E}(\mathcal{G})$ and $\mathcal{E}\left(\mathcal{T}_{G}\right)$ are equal.

\section{The $G$-descents to $G$-peaks map}

\subsection{The $\Theta_{G}$-function}

There is a well known Hopf endomorphism $\Theta$ on symmetric functions whose image is the space spanned by $Q$-Schur functions [19]. Several authors $[1,2,8,28]$ have extended this morphism to the quasi-symmetric functions, Hopf algebras and $(\Sigma, *, \Delta)$. It plays an important role in studying the peak algebra. We first recall the definition of $\Theta: \Sigma \rightarrow \Sigma$.

We have that $\Sigma=\mathcal{E}(D)=\bigoplus_{n \geq 0} \mathbb{k} \otimes_{\mathbb{Z}} \Sigma_{n}$, where $\Sigma_{n}$ is the free $\mathbb{Z}$-module spanned by $\left\{d_{\mathbf{c}} \mid \mathbf{c}=n\right\}$ (see Section 1.2). It is well known that $\Sigma$ is freely generated as an algebra by $\left\{d_{(n)}=1_{n} \mid n \geq 1\right\}$ (see $[17,18,29]$ ). We set

$$
\Theta\left(d_{(n)}\right)=2 \stackrel{\circ}{p}_{(n)}
$$

This defines a unique morphism of algebras, and it is straightforward to check that $\Theta\left(\Delta\left(d_{(n)}\right)\right)=\Delta\left(\stackrel{\circ}{p}_{(n)}\right)$. Hence $\Theta: \Sigma \rightarrow \Sigma$ is a Hopf morphism whose image is $\stackrel{\circ}{\mathcal{P}}$. It is exactly the morphism defined in $[1,2,8,28]$. 
For any $\mathbf{c}=n$, there is an explicit formula for $\Theta\left(d_{\mathbf{c}}\right)$ (see for example [1, Theorem 5.8]). For this we need to introduce more notation on compositions. For $\mathbf{c}=\left(c_{1}, c_{2}, \ldots c_{s}\right) \models n$ we denote the number of parts of $\mathbf{c}$ by $\tau(\mathbf{c})=s$. There is a natural map from compositions of $n$ to peak compositions. For $\mathbf{c}=\left(c_{1}, c_{2}, \ldots c_{s}\right) \models n$, let $\Lambda(\mathbf{c}) \in \stackrel{\circ}{\Pi}_{n}$ be the peak composition obtained from $\mathbf{c}$ as follows. The composition $\mathbf{c}$ factorizes uniquely into compositions $\mathbf{c}_{1} \cdot \mathbf{c}_{2} \cdots \mathbf{c}_{r}$ where for $i<r$ we have $\mathbf{c}_{i}=(1,1, \ldots, 1, m)$ and $m>1$, and $\mathbf{c}_{r}=(1,1, \ldots, 1)$. In this factorization, any sequence of 1 's may be empty. We then define

$$
\Lambda(\mathbf{c})=\left(\left\|\mathbf{c}_{1}\right\|,\left\|\mathbf{c}_{2}\right\|, \ldots,\left\|\mathbf{c}_{r}\right\|\right) \in \stackrel{\circ}{\Pi}_{n},
$$

removing the last part if it is zero. For example,

$$
\Lambda((1,2,1,1,3,3))=(\|(1,2)\|,\|(1,1,3)\|,\|(3)\|)=(3,5,3) .
$$

Similarly, $\Lambda((2,1,3,1))=(2,4,1)$, and $\Lambda((2,1,1))=(2,2)$. Finally, recall from Eq. (1) that $I_{\mathbf{c}}$ is a subset of $[1, n-1]$. For any $\mathbf{c} \models n$,

$$
\Theta\left(d_{\mathbf{c}}\right)=\sum_{\mathbf{e} \in \Phi(\mathbf{c})} 2^{\tau(\Lambda(\mathbf{e}))} d_{\mathbf{e}}
$$

where

$$
\Phi(\mathbf{c})=\left\{\mathbf{e}=\|\mathbf{c}\| \mid\left(i \in I_{\Lambda(\mathbf{e})} \Rightarrow\left|\{i-1, i\} \cap I_{\mathbf{c}}\right|=1\right)\right\} .
$$

Since $D$ satisfies (FP) and (IP), Corollaries 3.8 and 3.9 give that the assignment $G \rightsquigarrow \mathcal{E}\left(D_{G}\right)$ is functorial and $\Sigma(G)=\mathcal{E}\left(D_{G}\right)$ is freely generated as an algebra by

$$
\left\{d_{(n)^{g}}^{G}=\left(1_{n}\right)^{g} \mid n \geq 1, g \in G\right\} .
$$

Proposition 5.1. The map $\Theta_{G}: \Sigma(G) \rightarrow \Sigma(G)$ defined by

$$
\Theta\left(d_{(n)^{g}}^{G}\right)=2 \stackrel{\circ}{(n) g}^{g}
$$

is a Hopf morphism. Moreover, $\Theta_{G}(\Sigma(G))=\stackrel{\circ}{\mathcal{P}}(G)$.

Proof: Equation (38) defines a unique morphism of algebras. It is straightforward to check that $\Theta\left(\Delta\left(d_{(n)^{g}}^{G}\right)\right)=\Delta\left(\stackrel{\circ}{p}_{(n)^{g}}^{G}\right)$. Hence $\Theta_{G}$ is a Hopf morphism whose image, by Theorem 4.1, is $P(G)$.

For any $G$-composition $\mathbf{c}^{\mathbf{g}}$, we are interested in an explicit formula for $\Theta_{G}\left(d_{\mathbf{c}^{\mathrm{g}}}^{G}\right)$. For this, let $\mathbf{c}^{\mathbf{g}}=\mathbf{c}_{1}{ }^{g_{1}} \cdot \mathbf{c}_{1}{ }^{{ }^{2}} \cdots \mathbf{c}_{k}{ }^{{ }^{g} k}$ be the rainbow decomposition of $\mathbf{c}^{\mathrm{g}}$. From Corollary 3.8 we clearly have that

$$
d_{\mathbf{c}^{\mathbf{g}}}^{G}=d_{\mathbf{c}_{1} g_{1}}^{G} * \cdots * d_{\mathbf{c}_{k} g_{k}}^{G}=\left(d_{\mathbf{c}_{1}}^{G}\right)^{g_{1}} * \cdots *\left(d_{\mathbf{c}_{k}}^{G}\right)^{g_{k}} .
$$


Now, from Eq. (38), it is also clear that for a single $g \in G$, we have

$$
\Theta\left(d_{\mathbf{c}^{g}}^{G}\right)=\Theta\left(\left(d_{\mathbf{c}}^{G}\right)^{g}\right)=\left(\Theta\left(d_{\mathbf{c}}^{G}\right)\right)^{g} .
$$

Combining this with Eq. (36) we obtain the following theorem.

Theorem 5.2. Let $G$ be a finite abelian group and let $\mathbf{c}^{\mathbf{g}}=\mathbf{c}_{1}{ }^{g_{1}} \cdot \mathbf{c}_{2}{ }^{g_{2}} \cdots \mathbf{c}_{k}{ }^{g_{k}}$ be the rainbow decomposition of the $G$-composition $\mathbf{c}^{\mathbf{g}}$. We have

$$
\Theta_{G}\left(d_{\mathbf{c}^{\mathrm{g}}}^{G}\right)=\sum_{1 \leq i \leq k, \mathbf{e}_{i} \in \Phi\left(\mathbf{c}_{i}\right)} 2^{\sum_{i=1}^{k} \tau\left(\Lambda\left(\mathbf{e}_{i}\right)\right)} d_{\mathbf{e}_{1} g_{1} \cdot \mathbf{e}_{2} g_{2} \ldots \mathbf{e}_{k} g_{k}}^{G} .
$$

\subsection{The dual side}

Let $\mathcal{A}$ be a countable alphabet viewed as a set of variables and $G$ be a finite abelian group. We let $\mathcal{Z}=\mathcal{A}^{G}$ be the alphabet where we put an arbitrary total order on $G$ and we order $\mathcal{Z}$ as follows. For $a^{g}, b^{h} \in \mathcal{Z}$, we set $a^{g}<b^{h}$ if and only if $(a<b)$ or $(a=b$ and $g<h)$. In this section we consider subspaces of the space $\mathbb{k}[[\mathcal{Z}]]$ of formal series in the variables $a^{g} \in \mathcal{Z}$.

Let $\mathbf{c}^{\mathbf{g}}=\left(c_{1}^{g_{1}}, c_{2}^{g_{2}}, \ldots, c_{k}^{g_{k}}\right)$ be a $G$-composition of $n$ and set $t_{i}=c_{1}+c_{2}+\cdots+c_{i}$ for each $i$. We denote by

$$
\left(h_{1}, h_{2}, \ldots, h_{n}\right)=(\underbrace{g_{1}, g_{1}, \ldots, g_{1}}_{c_{1} \text { times }}, \underbrace{g_{2}, g_{2}, \ldots, g_{2}}_{c_{2} \text { times }}, \ldots, \underbrace{g_{k}, g_{k}, \ldots, g_{k}}_{c_{k} \text { times }}) .
$$

We let $\mathcal{Z}_{\mathbf{c}^{\mathrm{g}}}$ be the set of all $n$-tuples $\left(z_{1}, z_{2}, \ldots, z_{n}\right) \in \mathcal{Z}^{n}$ where $z_{i}=a_{i}^{h_{i}}$ for some $a_{1} \leq a_{2} \leq \cdots \leq a_{n}$ such that

$$
\forall i \in\{1,2, \ldots, k-1\}, \quad g_{i} \geq g_{i+1} \Longrightarrow a_{t_{i}}<a_{t_{i}+1}
$$

Finally, we define the formal series in $\mathbb{k}[[\mathcal{Z}]]$

$$
F_{\mathbf{c}^{\mathrm{g}}}=\sum_{\left(z_{1}, z_{2}, \ldots, z_{n}\right) \in \mathcal{Z}_{\mathbf{c}^{\mathrm{g}}}} z_{1} z_{2} \cdots z_{n}
$$

In [6] the authors have shown that the vector subspace $\operatorname{QSym}(G)$ spanned by the $F_{\mathbf{c}^{\text {g }}}$ for all $G$-compositions is a subalgebra of $\mathbb{k}\left[\mathcal{A}^{G}\right]$. It is a graded connected Hopf algebra that is graded dual to $\Sigma(G)$. In particular, the basis $\left\{F_{\mathbf{c}^{\mathrm{g}}}\right\}$ of $\operatorname{QSym}(G)$ is dual to the basis $\left\{d_{\mathbf{c}^{g}}^{G}\right\}$ of $\Sigma(G)$. To see this, we first recall that $\mathbb{k}[G]$ is self dual. If $\alpha \in G_{n}$, then $\alpha^{-1}=\alpha^{*}$ is its dual element in the dual basis [6, Section 5.1]. For any $G$-composition c, $\alpha^{*}\left(d_{\mathbf{c}}^{G}\right)=1$ iff $D_{G}\left(\alpha^{-1}\right)=\mathbf{c}$. But by [6, Proposition 22 and Diag. (11)] we know that the dual of $\Sigma(G)$ is obtained as the quotient of $\mathbb{k}[G]$ by the relation $\alpha \sim_{R_{G}} \beta$ where $R_{G}(\alpha)=D_{G}\left(\alpha^{-1}\right)$. For $\alpha \in G_{n}$ such that $R_{G}(\alpha)=\mathrm{c}$ we have from [6, Theorem 33 and Proposition. 34] that the image of $\alpha^{*}$ in this quotient corresponds to $F_{\mathbf{c}}$ of QSym $(G)$. We then have that $F_{\mathbf{c}}\left(d_{\mathbf{e}}^{G}\right)=1$ if and only if $\mathbf{c}=\mathbf{e}$. 
For a $G$-composition $\mathbf{c}^{\mathbf{g}}$ let $\mathbf{c}^{\mathbf{g}}=\mathbf{c}_{(1)}{ }^{g_{1}} \cdot \mathbf{c}_{(2)}{ }^{g_{2}} \cdots \mathbf{c}_{(k)}{ }^{g_{k}}$ be its rainbow decomposition and let $n_{i}=\left\|\mathbf{c}_{(i)}\right\|$. Using Eq. (39), the morphism $\Theta_{G}$ has a graded dual $\Theta_{G}^{*}: \operatorname{QSym}(G) \rightarrow \operatorname{QSym}(G)$ given by

$$
\Theta_{G}^{*}\left(F_{\mathbf{c}^{\mathrm{g}}}\right)=2^{\sum_{i=1}^{k} \tau\left(\Lambda\left(\mathbf{c}_{i}\right)\right)} \sum_{1 \leq i \leq k, \mathbf{e}_{i} \in \Phi^{*}\left(\mathbf{c}_{i}\right)} F_{\mathbf{e}_{1} g_{1} \cdot \mathbf{e}_{2} g_{2} \ldots \mathbf{e}_{k} g_{k}},
$$

where

$$
\Phi^{*}(\mathbf{c})=\left\{\mathbf{e} \models\|\mathbf{c}\| \mid\left(i \in I_{\Lambda(\mathbf{c})} \Rightarrow\left|\{i-1, i\} \cap I_{\mathbf{e}}\right|=1\right)\right\} .
$$

We deduce the following theorem

Theorem 5.3. The image of $\Theta_{G}^{*}$ is the graded dual Hopf algebra $\stackrel{\circ}{\mathcal{P}}(G)^{*}$ of $\stackrel{\circ}{\mathcal{P}}(G)$.

\section{3. $G$-coloured combinatorial Hopf algebras}

In [2] we introduced the theory of combinatorial Hopf algebras and associated objects. The framework consists of pairs $(\mathcal{H}, \zeta)$ where $\mathcal{H}$ is a connected graded Hopf algebra and $\zeta: \mathcal{H} \rightarrow \mathbb{k}$ is an algebra morphism (called its character). We say that $(\mathcal{H}, \zeta)$ is odd if for any homogeneous element $x \in \mathcal{H}$ of degree $n$ we have $\zeta \circ S(x)=(-1)^{n} \zeta(x)$ where $S$ is the antipode of $\mathcal{H}$. We have shown in that paper that for a certain character $\zeta_{\mathcal{Q}}$ the pair $\left(\mathrm{QSym}, \zeta_{\mathcal{Q}}\right)$ is the terminal object for the category of pairs $(\mathcal{H}, \zeta)$. Moreover we have shown that $\mathcal{\mathcal { P }}$ is the so-called odd subalgebra of $\left(\mathrm{QSym}, \zeta_{\mathcal{Q}}\right)$. These algebras play an important role among the connected graded Hopf algebras and we refer the reader to [2] for more motivation. Here we are interested in a similar theory for the $G$ coloured version. We will just outline the ideas, as this should be part of forthcoming work.

For $G=\left\{1^{G}\right\}$, The map $\zeta_{\mathcal{Q}}$ : QSym $\rightarrow \mathbb{k}$ is the morphism of algebras defined by $\zeta_{\mathcal{Q}}(F)=F(1,0,0, \ldots)$. The functoriality of the construction of $\operatorname{QSym}(G)$ implies that the following diagram commutes

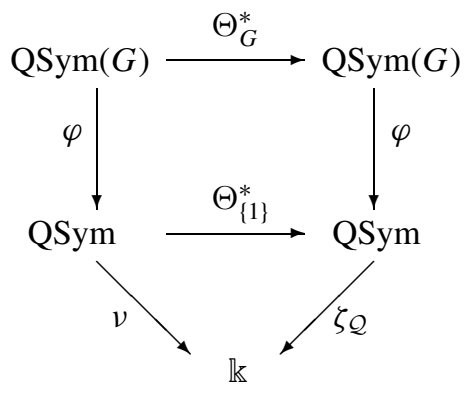

where $v=\zeta_{\mathcal{Q}} \circ \Theta_{\left\{1^{G}\right\}}^{*}$ and $\varphi$ is the Hopf morphism induced by the inclusion $\left\{1^{G}\right\} \rightarrow$ $G$. We have shown in [2, Proposition 6.4] that the pair (QSym $(\{1\}), v)$ is odd, hence 祭Springer 
$(\operatorname{QSym}(G), v \circ \varphi)$ is odd. Using [2, Proposition 6.1] in such a situation gives us that the image of $\Theta_{G}^{*}$, that is $\stackrel{\circ}{\mathcal{P}}(G)^{*}$, must be contained in the odd subalgebra of QSym $(G)$. But a simple dimension count gives us that the odd subalgebra of $\left(\operatorname{QSym}(G), \zeta_{\mathcal{Q}} \circ \varphi\right)$ is larger than $\stackrel{\triangleright}{\mathcal{P}}(G)^{*}$. On the other hand, if we restrict ourselves on a smaller category, then an interesting theory unfolds.

Let $G$ be a fixed finite abelian group. Consider pairs $\left(\mathcal{H}, \zeta^{G}\right)$ where $\mathcal{H}$ is a connected graded Hopf algebra and $\zeta: \mathcal{H} \rightarrow \mathbb{k}[G]$ is an algebra morphism. In analogy with [2] we will call such a morphism $\zeta: \mathcal{H} \rightarrow \mathbb{k}[G]$ a $G$-character. The category of combinatorial $G$-coloured Hopf algebras consists of pairs $\left(\mathcal{H}, \zeta^{G}\right)$ as above and graded Hopf morphisms $\Psi$ such that

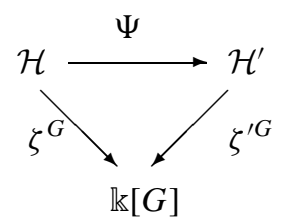

commutes.

There is a natural algebra morphism $\mathbb{k}\left[\mathcal{A}^{G}\right] \rightarrow \mathbb{k}[G]$ which maps the variable $a_{i}^{g} \in \mathcal{A}^{G}$ to $g$ if $i=1$ and to zero otherwise. This gives us the algebra morphism $\zeta_{\mathcal{Q}}^{G}: \operatorname{QSym}(G) \rightarrow \mathrm{QSym}(G)$ where

$$
\zeta_{\mathcal{Q}}^{G}\left(F_{\mathbf{c}^{\mathbf{g}}}\right)= \begin{cases}g & \text { if } \mathbf{c}=(n) \text { and } \mathbf{g}=g, \\ 1 & \text { if } \mathbf{c}=(), \\ 0 & \text { otherwise }\end{cases}
$$

We have a theorem very similar to [2, Theorem 4.1] with essentially the same proof.

Theorem 5.4. The pair $\left(\mathrm{QSym}(G), \zeta_{\mathcal{Q}}^{G}\right)$ is the terminal object in the category of combinatorial G-coloured Hopf algebras.

We then define the convolution product of two $G$-characters as follows. For two $G$-characters $\zeta^{G}, v^{G}: \mathcal{H} \rightarrow \mathbb{k}[G]$ let

$$
\zeta^{G} * \nu^{G}=m_{\mathbb{k}[G]} \circ\left(\zeta^{G} \otimes v^{G}\right) \circ \Delta_{\mathcal{H}}
$$

$G$-characters form a group with inverse (for the convolution) given by $\left(\zeta^{G}\right)^{-1}=$ $\zeta^{G} \circ S_{\mathcal{H}}$ where $S_{\mathcal{H}}$ is the antipode of $\mathcal{H}$. For a $G$-character $\zeta^{G}: \mathcal{H} \rightarrow \mathbb{k}[G]$ we let $\overline{\zeta^{G}}: \mathcal{H} \rightarrow \mathbb{k}[G]$ be the character such that for a homogeneous element $h \in \mathcal{H}$ of degree $n, \quad \overline{\zeta^{G}}(h)=(-1)^{n} \zeta^{G}(h)$. The odd $G$-subalgebra $S_{-}\left(\mathcal{H}, \zeta^{G}\right)$ of a pair $\left(\mathcal{H}, \zeta^{G}\right)$ is defined as in [2, Definition 5.7], namely the largest graded subcoalgebra of $\mathcal{H}$ such that for all $h \in S_{-}\left(\mathcal{H}, \zeta^{G}\right)$ we have $\overline{\zeta^{G}}(h)=\left(\zeta^{G}\right)^{-1}(h)$. It is in this context that

$$
S_{-}\left(\operatorname{QSym}(G), \zeta_{\mathcal{Q}}^{G}\right)=P(G)^{*}
$$


Combining this with an analog of $\left[2\right.$, Corollary 6.2], we have that $\left(\stackrel{\circ}{\mathcal{P}}(G)^{*}, \zeta_{\mathcal{Q}}^{G}\right)$ is the terminal object of odd combinatorial $G$-coloured Hopf algebras.

\section{References}

1. M. Aguiar, N. Bergeron, and K. Nyman, "The peak algebra and the descent algebras of type B and D," Trans. of the A.M.S. 356(7) (2004), 2781-2824.

2. M. Aguiar, N. Bergeron, and F. Sottile, "Combinatorial Hopf algebras and generalized DehnSommerville relations," Compositio Mathematica 142(1) (2006), 1-30.

3. M. Aguiar, K. Nyman, and R. Orellana, "New results on the peak algebra," J. Algebraic Comb. 23 (2006), 149-188. DOI: 10.1007/s10801-006-6922-8

4. M. Aguiar and H. Thomas, private communications (2004).

5. M.D. Atkinson, "A new proof of a theorem of Solomon," Bull. London Math. Soc. 18 (1986), 351-354.

6. P. Baumann and C. Hohlweg, A Solomon Theory for the Wreath Products $G$ ? $\mathfrak{S}_{n}$, preprint (2005), To appear in Trans. of the AMS.

7. F. Bergeron, N. Bergeron, R.B. Howlett, and D.E. Taylor, "A decomposition of the descent algebra of a finite Coxeter group," J. Alg. Combin. 1 (1992), 23-44.

8. N. Bergeron, F. Hivert, and J.Y. Thibon, "The peak algebra and the Hecke-Clifford algebras at $q=0$," J. Combin. Theory, Ser. A 107(1) (2004), 1-19.

9. N. Bergeron, S. Mykytiuk, F. Sottile, and S. van Willigenburg, "Pieri operations on posets," J. Combin. Theory, Ser. A 91 (2000), 84-110.

10. A. Björner and M. Wachs, "Generalized quotients in Coxeter groups," Trans. Amer. Math. Soc. 308 (1988), 1-37.

11. V.V. Deodhar, "Some characterizations of Bruhat ordering on a Coxeter group and determination of the relative Möbius function," Inv. Math. 39 (1977), 187-198.

12. F. Hivert, J.-C. Novelli, and J.-Y. Thibon, "Un analogue du monoïde plaxique pour les arbres binaires de recherche," C. R. Acad. Sci. Paris Sér I Math. 332 (2002), 577-580.

13. F. Hivert, J.-C. Novelli, and J.-Y. Thibon, "Sur quelques propriétés de l'algèbre des arbres binaires," $C$. R. Acad. Sci. Paris Sér I Math. 337 (2003), 565-568.

14. F. Hivert, J.C. Novelli, and J.Y. Thibon, The Algebra of Binary Search Trees, ArXiv preprint math. CO/0401089, to appear in Theoretical Computer Science (2004).

15. F. Hivert, J.-C. Novelli, and J.-Y. Thibon, "Representation theory of the 0-Ariki-Koike-Shoji algebras," preprint arXiv:math.CO/0407218.

16. J.E. Humphreys, Reflection Groups and Coxeter Groups, vol. 29, Cambridge University Press, 1990.

17. J.-L. Loday and M. Ronco, "Hopf algebra of the planar binary trees," Adv. Math. 139 (1998), 293-309.

18. C. Malvenuto and C. Reutenauer, "Duality between quasi-symmetric functions and Solomon descent algebra," J. Algebra 177 (1995), 967-982.

19. I. Macdonald, Symmetric Functions and Hall Polynomials, 2nd edition, Oxford Univ. Press, 1995.

20. R. Mantaci and C. Reutenauer, "A generalization of Solomon's algebra for hyperoctahedral groups and other wreath products," Comm. in Algebra 23(1) (1995), 27-56.

21. K. Nyman, "The peak algebra of the symmetric group,” J. Alg. Combin. 17 (2003), 309-322.

22. J.-C. Novelli and J.-Y. Thibon, "Free quasi-symmetric functions of arbitrary level," preprint arXiv:math.CO/0405597 (2004).

23. S. Poirier and C. Reutenauer, "Algèbres de Hopf de tableaux," Ann. Sci. Math. Québec 19 (1995), 79-90.

24. N. Reading, Cambrian Lattices, Adv. in Math. CO/0402086 (2004).

25. V. Reiner, "Equivariant fiber polytopes," Doc. Math. 7 (2002), 113-132 (electronic).

26. M. Schocker, "The peak algebra of the symmetric group revisited," Advances in Math. (to appear).

27. L. Solomon, "A Mackey formula in the group ring of a Coxeter group," J. Algebra 41 (1976), 255-268.

28. J.R. Stembridge, "Enriched P-partitions," Trans. Amer. Math. Soc. 349 (1997), 763-788.

29. J.-Y. Thibon, "Lectures on noncommutative symmetric functions," in Interaction of Combinatorics and Representation Theory, pp. 39-94. Math. Soc. Japan Memoirs 11, Tokyo: Math. Soc. Japan, 2001.

30. J. Tits, "Buildings of spherical type and finite BN-pairs," Lecture Notes in Mathematics 386 (1974). 